\title{
Crystal Bases for Quantum Classical Algebras and Nakajima's Monomials ${ }^{\dagger}$
}

\author{
By \\ Seok-Jin KANG*, Jeong-Ah KIM** and Dong-Uy SHIN*
}

\begin{abstract}
Using Nakajima's monomials, we construct a new realization of crystal bases for finite dimensional irreducible modules over quantum classical algebras. We also give an explicit bijection between the monomial realization and the Young tableau realization of crystal bases.
\end{abstract}

\section{Introduction}

In [5], Kashiwara developed the crystal basis theory, which initiated a vast variety of research in combinatorial representation theory. Crystal bases can be understood as bases at $q=0$, and they reflect the internal structure of integrable modules over quantum groups. Therefore, it is a very natural and fundamental problem to find explicit realizations of irreducible highest weight crystals $B(\lambda)$ over quantum groups.

For classical Lie algebras, Kashiwara and Nakashima gave an explicit realization of crystal bases for finite dimensional irreducible modules in terms of semistandard Young tableaux with given shapes satisfying certain additional

Communicated by M. Kashiwara. Received November 6, 2003.

2000 Mathematics Subject Classification(s): 81R50, 20 G05.

${ }^{\dagger}$ This article is an invited contribution to a special issue of Publications of RIMS commemorating the fortieth anniversary of the founding of the Research Institute for Mathematical Sciences.

* This research was supported by KOSEF Grant \# R01-2003-000-10012-0 and KRF Grant \# 2003-070-C00001.

School of Mathematics, Korea Institute for Advanced Study, Seoul 130-722, Korea.

e-mail: sjkang@kias.re.kr, shindong@kias.re.kr

** This research was supported by KOSEF Grant \# R01-2003-000-10012-0 and BK21

Mathematical Sciences Division, Seoul National University.

Department of Mathematics, Seoul National University, Seoul 151-747, Korea.

e-mail: jakim@math.snu.ac.kr

(C) 2004 Research Institute for Mathematical Sciences, Kyoto University. All rights reserved. 
conditions [7]. In [3], Kang, Kim, Lee and Shin gave another realization of crystal bases for classical Lie algebras using combinatorics of Young walls which were introduced in [2]. In [10], while studying the connection between the Kashiwara-Nakashima realization and the Young wall realization, Kim and Shin found another realization of crystal bases: the irreducible highest weight crystal $B(\lambda)$ for a classical Lie algebra can be identified with the set of semistandard reverse Young tableaux with a given shape. The reverse Young tableaux can be constructed from the Kashiwara-Nakashima tableaux by the bumping procedure.

On the other hand, in [11], Nakajima discovered that one can define a crystal structure on the set of irreducible components of a lagrangian subvariety $\mathfrak{Z}$ of the quiver variety $\mathfrak{M}$. In [12], while studying the $t$-analogs of $q$-characters of standard modules, Nakajima showed that these irreducible components can be identified with certain monomials and that the action of Kashiwara operators can be interpreted as multiplication by monomials. Moreover, in [6, 12], Kashiwara and Nakajima showed that the connected component $\mathcal{M}(\lambda)$ of $\mathcal{M}$ containing a maximal weight vector with a dominant integral weight $\lambda$ is isomorphic to the irreducible highest weight crystal $B(\lambda)$. However, it is still an open problem to find explicit characterizations of the monomials in $\mathcal{M}(\lambda)$ for general symmetrizable Kac-Moody algebras.

In this paper, we give an explicit characterization of the monomials in $\mathcal{M}(\lambda)$ for classical Lie algebras. Since the case of $\mathfrak{g}=A_{n}$ was worked out in [4], we will focus on the case when $\mathfrak{g}=C_{n}, B_{n}$ and $D_{n}$. We will also construct explicit bijections between the monomial realization, the reverse Young tableau realization and the Kashiwara-Nakashima tableau realization of crystal bases.

For the quantum affine algebras of type $A_{n}^{(1)}$, Kim gave an explicit characterization of the monomials in $\mathcal{M}(\lambda)$ [8]. We expect one can generalize this method to the other classical quantum affine algebras.

\section{$\S 1$. Nakajima's Monomials and Crystals}

The basic notions on quantum groups and crystal bases may be found in $[1,5]$. In this section, we briefly explain the crystal structure on the set of monomials discovered by Nakajima [12] following the exposition given in $[6]$. 
Let us fix the basic notations :

$\mathfrak{g}$ : classical finite dimensional simple Lie algebra,

$U_{q}(\mathfrak{g})$ : quantum classical algebra,

$I$ : index set for the simple roots of $\mathfrak{g}$,

$P^{\vee}=\bigoplus_{i \in I} \mathbf{Z} h_{i}:$ dual weight lattice,

$\alpha_{i}, \Lambda_{i}(i \in I)$ : simple roots, fundamental weights.

$P=\left\{\lambda \in \mathfrak{h}^{*} \mid \lambda\left(P^{\vee}\right) \subset \mathbf{Z}\right\}$ : weight lattice,

$P^{+}=\left\{\lambda \in P \mid \lambda\left(h_{i}\right) \geq 0\right.$ for all $\left.i \in I\right\}$ : the set of dominant integral weights, $\tilde{e}_{i}, \tilde{f}_{i}(i \in I):$ Kashiwara operators.

Let $\mathcal{M}$ be the set of monomials in the commuting variables $Y_{i}(n)(i \in I$, $n \in \mathbf{Z}$ ) and let

$$
M=Y_{i_{1}}\left(n_{1}\right)^{a_{1}} \cdots Y_{i_{r}}\left(n_{r}\right)^{a_{r}}, \quad\left(i_{k} \in I, n_{k}, a_{k} \in \mathbf{Z}\right)
$$

be an element on $M$. We define

$$
\begin{aligned}
\operatorname{wt}(M) & =\sum_{k=1}^{r} a_{k} \Lambda_{i_{k}}=a_{1} \Lambda_{i_{1}}+\cdots a_{r} \Lambda_{i_{r}}, \\
\varphi_{i}(M) & =\max \left(\left\{\sum_{\substack{k=1 \\
i_{k}=i}}^{s} a_{k} \mid 1 \leq s \leq r\right\} \cup\{0\}\right), \\
\varepsilon_{i}(M) & =\max \left(\left\{-\sum_{\substack{k=s+1 \\
i_{k}=i}}^{r} a_{k} \mid 1 \leq s \leq r-1\right\} \cup\{0\}\right), \\
n_{f} & =\text { the smallest } n_{s} \text { such that } \varphi_{i}(M)=\sum_{\substack{k=1 \\
i_{k}=i}}^{s} a_{k}, \\
n_{e} & =\text { the largest } n_{s} \text { such that } \varphi_{i}(M)=\sum_{\substack{k=1 \\
i_{k}=i}}^{s} a_{k} .
\end{aligned}
$$

Also, choose a set $C=\left(c_{i j}\right)_{i \neq j}$ of integers such that $c_{i j}+c_{j i}=1$, and define

$$
A_{i}(n)=Y_{i}(n) Y_{i}(n+1) \prod_{j \neq i} Y_{j}\left(n+c_{j i}\right)^{\alpha_{i}\left(h_{j}\right)} .
$$


Then, the Kashiwara operators $\tilde{e}_{i}, \tilde{f}_{i}(i \in I)$ on $\mathcal{M}$ are defined by

$$
\tilde{f}_{i}(M)=\left\{\begin{array}{ll}
0 & \text { if } \varphi_{i}(M)=0, \\
A_{i}\left(n_{f}\right)^{-1} M & \text { if } \varphi_{i}(M)>0,
\end{array} \quad \tilde{e}_{i}(M)= \begin{cases}0 & \text { if } \varepsilon_{i}(M)=0, \\
A_{i}\left(n_{e}\right) M & \text { if } \varepsilon_{i}(M)>0 .\end{cases}\right.
$$

Proposition $1.1[6,12]$.

(a) The maps wt: $\mathcal{M} \rightarrow P, \varphi_{i}, \varepsilon_{i}: \mathcal{M} \rightarrow \mathbf{Z} \cup\{-\infty\}, \tilde{e}_{i}, \tilde{f}_{i}: \mathcal{M} \rightarrow \mathcal{M} \cup\{0\}$ define a $U_{q}(\mathfrak{g})$-crystal structure on $\mathcal{M}$

(b) Let $M_{0}$ be a monomial with weight $\lambda$ such that $\tilde{e}_{i} M_{0}=0$ for all $i \in I$, and let $\mathcal{M}(\lambda)$ be the connected component of $\mathcal{M}$ containing $M_{0}$. Then there exists a crystal isomorphism

$$
\mathcal{M}(\lambda) \longrightarrow B(\lambda) \text { given by } M_{0} \mapsto v_{\lambda},
$$

where $B(\lambda)$ is the irreducible highest weight crystal for $U_{q}(\mathfrak{g})$ with highest weight vector $v_{\lambda}$.

Example 1.2. Let $\mathfrak{g}=C_{2}$, and choose $c_{12}=1$ and $c_{21}=0$. Then the crystal $\mathcal{M}\left(\Lambda_{1}+\Lambda_{2}\right)$ is given as follows.

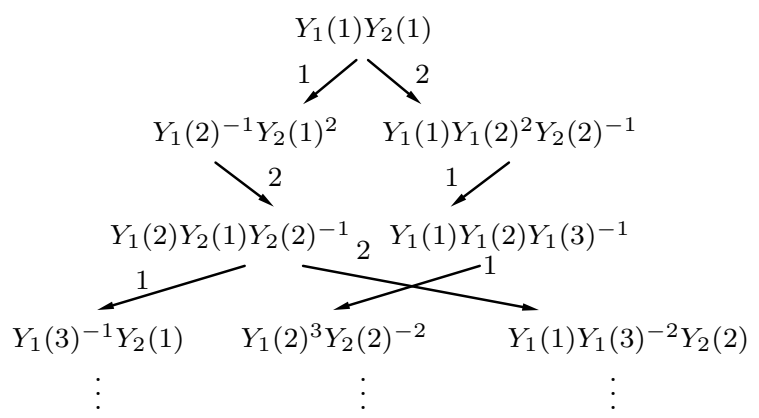

Note that $\mathcal{M}\left(\Lambda_{1}+\Lambda_{2}\right) \cong B\left(\Lambda_{1}+\Lambda_{2}\right)$.

In the next section, we will give an explicit characterization of the crystal $\mathcal{M}(\lambda)$ for the classical Lie algebras of type $B_{n}, C_{n}$, and $D_{n}$. For simplicity, we take

$$
c_{i j}= \begin{cases}0 & \text { if } i>j, \\ 1 & \text { if } i<j,\end{cases}
$$

and set $Y_{0}(m)^{ \pm 1}=1$ for all $m \in \mathbf{Z}$. 


\section{§2. Characterization of $\mathcal{M}(\lambda)$}

\section{$\S 2.1$. The $U_{q}\left(C_{n}\right)$-crystal $\mathcal{M}(\lambda)$}

Let $U_{q}\left(\mathfrak{s p}_{2 n}\right)$ be the quantum symplectic algebra. Then by (1.3), for $i \in I$ and $m \in \mathbf{Z}$, we have

$$
A_{i}(m)= \begin{cases}Y_{n-1}(m+1)^{-2} Y_{n}(m) Y_{n}(m+1) & \text { for } i=n \\ Y_{i-1}(m+1)^{-1} Y_{i}(m) Y_{i}(m+1) Y_{i+1}(m)^{-1} & \text { otherwise }\end{cases}
$$

We first consider the case when $\lambda=\Lambda_{k}(k \in I)$. Let $M_{0}=Y_{k}(m)$ for $m \in \mathbf{Z}$. Then by (1.2), it is easy to see that $\mathrm{w} t\left(M_{0}\right)=\Lambda_{k}$ and $\tilde{e}_{i} M_{0}=0$ for all $i \in I$. Hence, the connected component of $\mathcal{M}$ containing $M_{0}$ is isomorphic to $B\left(\Lambda_{k}\right)$ over $U_{q}(\mathfrak{g})$. For simplicity, we will take $M_{0}=Y_{k}(1)$.

Let $\mathbf{B}=\{1,2, \ldots, n, \overline{1}, \ldots, \bar{n}\}$ and define a total ordering on $\mathbf{B}$ by

$$
1 \prec 2 \prec \cdots \prec n \prec \bar{n} \prec \cdots \prec \overline{1} .
$$

We introduce new variables

$$
\begin{aligned}
& X_{i}(m)=Y_{i-1}(m+1)^{-1} Y_{i}(m), \\
& X_{\bar{i}}(m)=Y_{i-1}(m+(n-i+1)) Y_{i}(m+(n-i+1))^{-1}
\end{aligned}
$$

for $i, \bar{i} \in \mathbf{B}$ and $m \in \mathbf{Z}$. If $p-q=n-i(i=1, \ldots, n-1)$, it is straightforward verify that

$$
X_{i}(p) X_{\bar{i}}(q)=X_{i+1}(p) X_{\overline{i+1}}(q)
$$

Then we have the following characterization of the crystal $\mathcal{M}\left(\Lambda_{k}\right)$.

Proposition 2.1. For $k=1, \ldots, n$, let $M_{0}=Y_{k}(1)=X_{1}(k) \cdots X_{k}(1)$ be a maximal vector of weight $\Lambda_{k}$. Then the connected component $\mathcal{M}\left(\Lambda_{k}\right)$ of $\mathcal{M}$ containing $M_{0}$ is characterized as

$$
\mathcal{M}\left(\Lambda_{k}\right)=\left\{X_{i_{1}}(1) X_{i_{2}}(2) \cdots X_{i_{k}}(k) \mid \overline{1} \succeq i_{1} \succ i_{2} \succ \cdots \succ i_{k} \succeq 1\right\} .
$$

Proof. By Proposition 1.1, it suffices to prove the following statements: (C1) For all $i \in I$, we have $\tilde{e}_{i} \mathcal{M}\left(\Lambda_{k}\right) \subset \mathcal{M}\left(\Lambda_{k}\right) \cup\{0\}, \quad \tilde{f}_{i} \mathcal{M}\left(\Lambda_{k}\right) \subset \mathcal{M}\left(\Lambda_{k}\right) \cup$ $\{0\}$. 
(C2) For all $M \in \mathcal{M}\left(\Lambda_{k}\right)$, there exist a sequence of indices $i_{1}, \ldots, i_{t}$ in $I$ such that

$$
\tilde{e}_{i_{1}} \cdots \tilde{e}_{i_{t}} M=M_{0}
$$

Fix $i \in I$ and let $M=X_{i_{1}}(1) X_{i_{2}}(2) \cdots X_{i_{k}}(k) \in \mathcal{M}\left(\Lambda_{k}\right)$. Note that the expression of $M$ is not unique by $(2.4)$. However, if $\tilde{f}_{i} M \neq 0$, there exists $j \in\{1, \ldots, k\}$ such that $i_{j}=i$ or $\overline{i+1}$ and $i_{j+1}>i_{j}+1$ in any expression of $M$. Here, we use the notation

$$
i_{j}+1= \begin{cases}\overline{\overline{i_{j}}-1} & \text { for } i_{j} \in\{\overline{1}, \ldots, \bar{n}\} \\ \bar{n} & \text { for } i_{j}=n\end{cases}
$$

Observe that

$$
A_{i}(m)^{-1}=\left\{\begin{array}{lr}
X_{i}(m)^{-1} X_{i+1}(m)=X_{\overline{i+1}}(m-n+i)^{-1} X_{\bar{i}}(m-n+i) & \text { for } i \neq n, \\
X_{n}(m)^{-1} X_{\bar{n}}(m) & \text { for } i=n .
\end{array}\right.
$$

It follows that

$$
\tilde{f}_{i} M=\left\{\begin{array}{lll}
X_{i}^{-1}(j) X_{i+1}(j) M & \text { or } \quad X_{\overline{i+1}}^{-1}(j) X_{\bar{i}}(j) M & \text { for } i \neq n \\
X_{n}(j)^{-1} X_{\bar{n}}(j) M & \text { for } i=n
\end{array}\right.
$$

Similarly, one can prove $\tilde{e}_{i} M \in \mathcal{M}\left(\Lambda_{k}\right) \cup\{0\}$.

Let $M=X_{i_{1}}(1) X_{i_{2}}(2) \cdots X_{i_{k}}(k) \in \mathcal{M}\left(\Lambda_{k}\right)$. Suppose $\tilde{e}_{i} M=0$ for all $i \in I$. We will show that no $X_{\bar{a}}(p)$ appears in $M$. Then we can easily deduce that $M=X_{1}(k) \cdots X_{k}(1)=Y_{k}(1)$.

Suppose that for some $a(1 \leq a \leq n)$ and $p(1 \leq p \leq k), X_{\bar{a}}(p)=$ $Y_{a-1}(p+n-a+1) Y_{a}(p+n-a+1)^{-1}$ appears in $M$. Let $X_{\bar{\alpha}}(t)$ be the factor of $M$ such that $a=\max \left\{i \mid X_{\bar{a}}(p)\right.$ appears in $\left.M\right\}$. Then, since $\tilde{e}_{i} M=0$ for all $i \in I, X_{\alpha}(t+n-\alpha+1)=Y_{\alpha-1}(t+n-\alpha+2)^{-1} Y_{\alpha}(t+n-\alpha+1)$ appears in $M$. Moreover, we have

$$
\begin{aligned}
M & =X_{n-k+t+1}(k) \cdots X_{\alpha-1}(t+n-\alpha+2) X_{\alpha}(t+n-\alpha+1) X_{\bar{\alpha}}(t) M^{\prime} \\
& =X_{1}(k) \cdots X_{\alpha-1}(k-\alpha+2) X_{\alpha}(k-\alpha+1) X_{\bar{\alpha}}(t) M^{\prime} .
\end{aligned}
$$

Hence, $k=n+t$, which is a contradiction.

Remark 2.2. If we take $M_{0}=Y_{k}(N)$, then we need to replace $X_{i}(m)$ by $X_{i}(m+N-1)$.

Combining (2.4) and Proposition 2.1, we have 
Proposition 2.3. For $k=1, \ldots, n$, let $M_{0}=Y_{k}(1)=X_{1}(k) \ldots X_{k}(1)$ be a maximal vector of weight $\Lambda_{k}$. Then the connected component $\mathcal{M}\left(\Lambda_{k}\right)$ of $\mathcal{M}$ containing $M_{0}$ is characterized as

$$
\begin{aligned}
& \mathcal{M}\left(\Lambda_{k}\right)= \\
& \qquad \begin{array}{c}
(\mathrm{i}) \overline{1} \succeq i_{1} \succ i_{2} \succ \cdots \succ i_{k} \succeq 1 \\
X_{i_{1}}(1) X_{i_{2}}(2) \cdots X_{i_{k}}(k) ;\left(\text { ii) there is no } p, q \text { such that } i_{p}=a, i_{q}=\bar{a}\right\} . \\
\text { and } p-q=n-a .
\end{array}
\end{aligned}
$$

We now consider the general case.

Proposition 2.4. Let $\lambda=a_{1} \Lambda_{1}+\cdots+a_{n} \Lambda_{n}$. Then the connected component $\mathcal{M}(\lambda)$ containing the maximal vector

$$
M_{0}=Y_{1}(1)^{a_{1}} \cdots Y_{n}(1)^{a_{n}}=X_{1}(1)^{a_{1}}\left(X_{1}(2) X_{2}(1)\right)^{a_{2}} \cdots\left(X_{1}(n) \cdots X_{n}(1)\right)^{a_{n}}
$$

is characterized as the set of monomials

$$
M=X_{t_{1,1}}(1) \cdots X_{t_{1, \alpha_{1}}}(1) \cdots X_{t_{n, 1}}(n) \cdots X_{t_{n, \alpha_{n}}}(n)
$$

satisfying the following conditions:

(i) $\alpha_{k}=a_{k}+\cdots+a_{n}$ for $k=1, \ldots, n$,

(ii) $t_{k, 1} \succeq t_{k, 2} \succeq \cdots \succeq t_{k, \alpha_{k}}$ for $k=1, \ldots, n$,

(iii) for each $j=2, \ldots, n$ and $k=1, \ldots, \alpha_{j}, t_{j-1, k} \succ t_{j, k}$.

Proof. As in Proposition 2.1, it suffices to prove (C1) and (C2). Let $i \in I$ and $M$ be a monomial in $\mathcal{M}(\lambda)$. Assume that $\tilde{f}_{i} M \neq 0$. Then there exists $t_{j, k}=i$ or $\overline{i+1}$ for some $j$ and $k$ such that $t_{j-1, k}>t_{j, k}+1$ in any expression of $M$, and $\tilde{f}_{i} M$ is obtained from $M$ by multiplying

$$
\begin{array}{cc}
X_{i}^{-1}(j) X_{i+1}(j) \text { or } X_{\overline{i+1}}^{-1}(j) X_{\bar{i}}(j) & \text { for } i \neq n, \\
X_{n}(j)^{-1} X_{\bar{n}}(j) & \text { for } i=n .
\end{array}
$$

It is now easy to see that $\tilde{f}_{i} M \in \mathcal{M}(\lambda)$. Similarly, we can prove that $\tilde{e}_{i} \mathcal{M}(\lambda) \subset$ $\mathcal{M}(\lambda) \cup\{0\}$.

To prove (C2), suppose $M \in \mathcal{M}(\lambda)$ and $\tilde{e}_{i} M=0$ for all $i \in I$. Then by the same argument in the proof of Proposition 2.1, we can conclude $M=$ $Y_{1}(1)^{a_{1}} \cdots Y_{n}(1)^{a_{n}}$. 
Let $\lambda$ be a dominant integral weight and let $M$ be a monomial in $\mathcal{M}(\lambda)$. Then $M$ can be expressed as

$$
M=\prod_{i \in \mathbf{B} ; 1 \leq j \leq n} X_{i}(j)^{m_{i j}} .
$$

Unfortunately, by (2.4), this expression is not unique. However, one can find a canonical expression as is described in the following.

Step 1. Given an expression $M=\prod X_{i}(j)^{m_{i j}}$, we associate a tableau $T(M)$ as follows: for each $X_{i}(j)$, we put $i$ in the $j$-th row from the bottom in such a way that $i$ 's are weakly increasing in the $j$-th row. By the characterization of $\mathcal{M}(\lambda)$, the entries of $T(M)$ in each column are strictly increasing. For simplicity, we will say that there is an $i(p)$ if there exists an entry $i$ lying in the $p$-th row of $T(M)$ from the bottom.

Step 2. We define the following equivalence relations on the set of tableaux $T(M)$ :

(al-1) For each $a=1, \ldots, n-1$, if there is a pair $(a(p), \bar{a}(q))$ such that

(i) $p-q=n-a$, and $a(p)$ and $\bar{a}(q)$ lie in the same column, or

(ii) $\bar{a}(q)$ lies in the left hand side of $a(p)$,

then replace $(a(p), \bar{a}(q))$ with $(a+1(p), \overline{a+1}(q))$.

If there are several such pairs, then we carry out this process for the pair $(a(p), \bar{a}(q))$ consisting of the rightmost $a(p)$ and the leftmost $\bar{a}(q)$ and continue as is shown below.

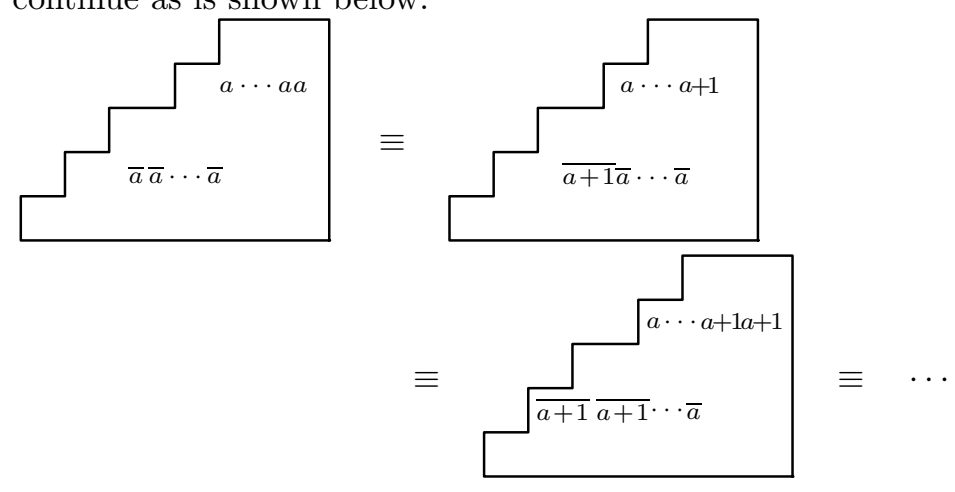

We will apply this rule from $a=1$ to $a=n-1$.

(al-2) For each $b=2, \ldots, n$, if there is a pair $(b(p), \bar{b}(q))$ such that $p-q=$ $n-b+1$, and $\bar{b}(q)$ lies in the right hand side of $b(p)$, then we replace 
$(b(p), \bar{b}(q))$ with $(b-1(p), \overline{b-1}(q))$. If there are several pairs $(b(p), \bar{b}(q))$, then we carry out this process for the pair $(b(p), \bar{b}(q))$ consisting of the leftmost $b(p)$ and the rightmost $\bar{b}(q)$ and continue as is shown below.

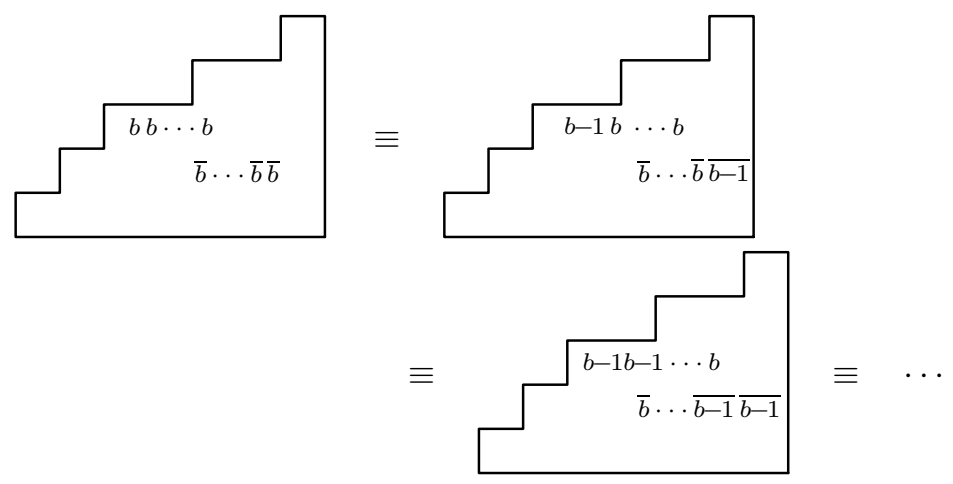

We will apply this rule from $b=n$ to $b=2$.

From now on, we will denote by $[T(M)]$ the tableau obtained from $T(M)$ by applying (al-1) and (al-2). The corresponding monomial $[M]$ will be called the canonical expression of $M$.

Remark 2.5. Note that (al-1) and (al-2) cannot occur simultaneously, and that $[M]$ does not depend on the order of (al-1) and (al-2).

Example 2.6. (a) Let $\lambda=\Lambda_{1}+2 \Lambda_{2}+\Lambda_{3}$ for $C_{3}$ and let $M=$ $Y_{1}(2)^{2} Y_{1}(3) Y_{1}(4)^{-2} Y_{2}(3)^{-1} Y_{3}(1)$. Then it can be expressed as

$$
M=X_{1}(3) X_{2}(2) X_{1}(2)^{2} X_{\overline{1}}(1)^{2} X_{\overline{2}}(1) X_{3}(1)
$$

and hence $M \in \mathcal{M}\left(\Lambda_{1}+2 \Lambda_{2}+\Lambda_{3}\right)$. Moreover, we have

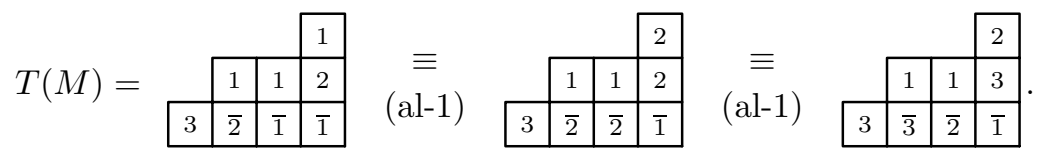

Therefore,

$$
[M]=X_{2}(3) X_{3}(2) X_{1}(2)^{2} X_{\overline{1}}(1) X_{\overline{2}}(1) X_{\overline{3}}(1) X_{3}(1) .
$$

(b) Let $\lambda=\Lambda_{2}+\Lambda_{3}$ for $C_{3}$ and let $M=Y_{1}(3) Y_{2}(2)^{2} Y_{2}(3)^{-2}$. Then it can be expressed as

$$
M=X_{1}(3) X_{3}(2)^{2} X_{\overline{3}}(1)^{2}
$$


and hence $M \in \mathcal{M}\left(\Lambda_{2}+\Lambda_{3}\right)$. Moreover, we have

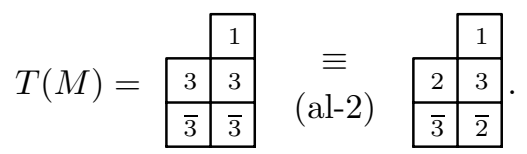

Therefore,

$$
[M]=X_{1}(3) X_{3}(2) X_{2}(2) X_{\overline{2}}(1) X_{\overline{3}}(1) .
$$

From the above algorithm, we have

Theorem 2.7. Let $\lambda=a_{1} \Lambda_{1}+\cdots+a_{n} \Lambda_{n}$. Then the connected component $\mathcal{M}(\lambda)$ containing the maximal vector $M_{0}$ given in Proposition 2.4 is characterized as the set of monomials

$$
M=X_{t_{1,1}}(1) \cdots X_{t_{1, \alpha_{1}}}(1) \cdots X_{t_{n, 1}}(n) \cdots X_{t_{n, \alpha_{n}}}(n)
$$

satisfying the following conditions:

(i) $\alpha_{k}=a_{k}+\cdots+a_{n}$ for $k=1, \ldots, n$,

(ii) $t_{k, 1} \succeq t_{k, 2} \succeq \cdots \succeq t_{k, \alpha_{k}}$ for $k=1, \ldots, n$,

(iii) for each $j=2, \ldots, n$ and $k=1, \ldots, \alpha_{j}, t_{j-1, k} \succ t_{j, k}$,

(iv) there is no pair $\left(X_{t_{p, k}}(p), X_{t_{q, l}}(q)\right)$ with $k \leq l, p>q$ such that

$$
t_{p, k}=a, t_{q, l}=\bar{a} \text { and } p-q=n-a,
$$

(v) there is no pair $\left(X_{t_{p, k}}(p), X_{t_{q, l}}(q)\right)$ with $k>l, p>q$ such that

$$
t_{p, k}=a, t_{q, l}=\bar{a} \text { and } p-q=n-a+1 .
$$

\section{§2.2. The $U_{q}\left(B_{n}\right)$-crystal $\mathcal{M}(\lambda)$}

Let $U_{q}\left(\mathfrak{s o}_{2 n+1}\right)$ be the quantum special orthogonal algebra. Then for $i \in I$ and $m \in \mathbf{Z}$, we have

$$
A_{i}(m)= \begin{cases}Y_{n-2}(m+1)^{-1} Y_{n-1}(m) Y_{n-1}(m+1) Y_{n}(m)^{-2} & \text { for } i=n-1, \\ Y_{i-1}(m+1)^{-1} Y_{i}(m) Y_{i}(m+1) Y_{i+1}(m)^{-1} & \text { otherwise }\end{cases}
$$


Set

$$
\omega_{i}= \begin{cases}\Lambda_{i} & \text { if } i \neq n \\ 2 \Lambda_{n} & \text { if } i=n .\end{cases}
$$

Then any dominant integral weight $\lambda$ can be expressed as

$$
\lambda=a_{1} \omega_{1}+\cdots+a_{n} \omega_{n}+b \Lambda_{n}
$$

where $a_{i} \in \mathbf{Z}_{\geq 0}(i=1, \ldots, n), b=0$ or 1 .

We first focus on the case when $\lambda=\omega_{k}(k=1, \ldots, n)$. As in $U_{q}\left(C_{n}\right)$ case, the connected component containing $M_{0}=Y_{k}(m)$ for $m \in \mathbf{Z}$ is isomorphic to $B\left(\omega_{k}\right)$. For simplicity, we will take $M_{0}=Y_{k}(1)$.

Let $\mathbf{B}=\{1,2, \ldots, n, 0, \overline{1}, \ldots, \bar{n}\}$ and define a total ordering on $\mathbf{B}$ by

$$
1 \prec 2 \prec \cdots \prec n \prec 0 \prec \bar{n} \prec \cdots \prec \overline{1} \text {. }
$$

For $m \in \mathbf{Z}$, we introduce new variables

$$
\begin{aligned}
& X_{i}(m)= \begin{cases}Y_{i-1}(m+1)^{-1} Y_{i}(m) & \text { for } i=1, \ldots, n-1, \\
Y_{n-1}(m+1)^{-1} Y_{n}(m)^{2} & \text { for } i=n,\end{cases} \\
& X_{\bar{i}}(m)= \begin{cases}Y_{i-1}(m+(n-i+1)) Y_{i}(m+(n-i+1))^{-1} & \text { for } i=1, \ldots, n-1, \\
Y_{n-1}(m+1) Y_{n}(m+1)^{-2} & \text { for } i=n,\end{cases} \\
& X_{0}(m)=Y_{n}(m) Y_{n}(m+1)^{-1} .
\end{aligned}
$$

Lemma 2.8. For $a, b, p, q=1, \ldots, n$ with $p>q$ and $i=1, \ldots, n-1$, we have

$$
\begin{aligned}
& X_{i}(p) X_{\bar{i}}(q)=X_{i+1}(p) X_{\overline{i+1}}(q) \quad \text { if } p-q=n-i, \\
& X_{0}(p)^{2}=X_{n}(p) X_{\bar{n}}(p), \\
& X_{0}(p) \sqrt{X_{a}(p) X_{\bar{n}}(p-1) \cdots X_{\overline{a+1}}(p-n+a)} \\
& =X_{a}(p) \sqrt{X_{\bar{n}}(p) \cdots X_{\overline{a+1}}(p-n+a+1) X_{\bar{a}}(p-n+a)}, \\
& X_{\bar{a}}(p) \sqrt{X_{a}(n-a+p) \cdots X_{n}(p)} \\
& =X_{0}(p) \sqrt{X_{a+1}(n-a+p) \cdots X_{n}(p+1) X_{\bar{a}}(p)}, \\
& X_{\bar{a}}(p) \sqrt{X_{a}(n-a+p) \cdots X_{b-1}(n-b+1+p)} \\
& \quad \times \sqrt{X_{b+1}(n-b+p) \cdots X_{n}(1+p) X_{\bar{b}}(p)}
\end{aligned}
$$




$$
\begin{aligned}
= & X_{\bar{b}}(p) \sqrt{X_{a+1}(n-a+p) \cdots X_{n}(1+p) X_{\bar{a}}(p)} \quad \text { if } a<b, \\
& X_{a}(p) \sqrt{X_{b}(p) X_{\bar{a}}(p-(n-a+1)) \cdots X_{\overline{b+1}}(p-n+b)} \\
= & X_{b}(p) \sqrt{X_{a}(p) X_{a-1}(p-(n-a+1)) \cdots X_{\bar{b}}(p-n+b)} \\
& \text { if } a>b .
\end{aligned}
$$

Then we have the following characterization of the crystal $\mathcal{M}\left(\omega_{k}\right)$.

Proposition 2.9. For $k=1, \cdots, n$, let $M_{0}=Y_{k}(1)$ be a maximal vector of weight $\omega_{k}$. Then the connected component $\mathcal{M}\left(\omega_{k}\right)$ of $\mathcal{M}$ containing $M_{0}$ is characterized as

$\mathcal{M}\left(\omega_{k}\right)=\left\{\begin{array}{l|l}X_{i_{1}}(1) X_{i_{2}}(2) \cdots X_{i_{k}}(k) & \begin{array}{l}\overline{1} \succeq i_{1} \succ i_{2} \succ \cdots \succ i_{k} \succeq 1, \text { but 0's can } \\ \text { be repeated }\end{array}\end{array}\right\}$.

Proof. The proof is similar to that of Proposition 2.1.

Combining Lemma 2.8 and Proposition 2.9, we obtain

Proposition 2.10. For $k=1, \ldots, n$, let $M_{0}=Y_{k}(1)$ be a maximal vector of weight $\omega_{k}$. Then the connected component $\mathcal{M}\left(\omega_{k}\right)$ of $\mathcal{M}$ containing $M_{0}$ is characterized as

$$
\begin{aligned}
& \mathcal{M}\left(\omega_{k}\right)= \\
& \left\{\begin{array}{l|l}
X_{i_{1}}(1) X_{i_{2}}(2) \cdots X_{i_{k}}(k) & \begin{array}{c}
\text { (i) } \overline{1} \succeq i_{1} \succ i_{2} \succ \cdots \succ i_{k} \succeq 1 \text {, but 0's can be } \\
\text { repeated, } \\
\text { (ii) there is no } p, q \text { such that } i_{p}=a, i_{q}=\bar{a}
\end{array}
\end{array}\right\} . \\
& \text { and } p-q=n-a \text {. }
\end{aligned}
$$

Proposition 2.11. Let $M_{0}=Y_{n}(1)$ be a maximal vector of weight $\Lambda_{n}$. Then the connected component $\mathcal{M}\left(\Lambda_{n}\right)$ of $\mathcal{M}$ containing $M_{0}$ is characterized as the set of monomials of the form

$$
\sqrt{X_{i_{1}}(1) X_{i_{2}}(2) \cdots X_{i_{n}}(n)}
$$

satisfying the following conditions:

(i) $\overline{1} \succeq i_{1} \succ i_{2} \succ \ldots \succ i_{n} \succeq 1$ and $i_{k} \neq 0$ for all $k=1, \ldots, n$,

(ii) there is no pair $\left(i_{p}=a, i_{q}=\bar{a}\right)$ for all $a, p, q=1, \ldots, n$ with $p>q$. 
Proof. Note that if $\tilde{f}_{i} M \neq 0(i \neq n)$, there are $\sqrt{X_{i}(p)}$ and $\sqrt{X_{\overline{i+1}}(q)}$ in $M$ with $p-q=n-i$ because of the conditions (i) and (ii). Moreover, in this case, $p-q=n-i$ and hence $\tilde{f}_{i} M$ is obtained from $M$ by multiplying $A_{i}(p)^{-1}$. That is, $\tilde{f}_{i} M$ is obtained from $M$ by replacing $\sqrt{X_{i}(p)}, \sqrt{X_{\overline{i+1}}(q)}$ with $\sqrt{X_{i+1}(p)}, \sqrt{X_{\bar{i}}(q)}$. Therefore, $\tilde{f}_{i} M \in \mathcal{M}\left(\Lambda_{n}\right)$.

Similarly, if $\tilde{f}_{n} M \neq 0$, then $\sqrt{X_{n}(p)}$ appears in $M$ and $\tilde{f}_{n} M$ is obtained from $M$ by multiplying $A_{n}(p)^{-1}$. That is, $\tilde{f}_{n} M$ is obtained from $M$ by replacing $\sqrt{X_{n}(p)}$ with $\sqrt{X_{\bar{n}}(p)}$. Therefore, $\tilde{f}_{n} M \in \mathcal{M}\left(\Lambda_{n}\right)$.

It remains to show that if $\tilde{e}_{i} M=0$ for all $i$, then $M=Y_{n}(1)$. Since $\tilde{e}_{n} M=0$, for some $p=1, \cdots, n, \sqrt{X_{n}(p)}$ must appear in $M$. Moreover, since $\tilde{e}_{n-1} M=0$ and $\sqrt{X_{n}(p)}$ appears in $M, \sqrt{X_{n-1}(p+1)}$ must appear in $M$. Hence $M$ must be $\sqrt{X_{1}(n) \cdots X_{n}(1)}=Y_{n}(1)$.

Remark 2.12. From the appearance, one may think $\sqrt{X_{i_{1}}(1) X_{i_{2}}(2) \cdots X_{i_{n}}(n)}$ may not be a monomial in $Y_{i}(k)$ 's. But, by the conditions (i) and (ii), all the $Y_{i}(k)$ 's appear even number of times in the expression. Hence it is a monomial in $Y_{i}(k)$ 's.

Now, we consider the general case.

Proposition 2.13. Let $\lambda=a_{1} \omega_{1}+\cdots+a_{n} \omega_{n}$ (resp. $a_{1} \omega_{1}+\cdots+a_{n} \omega_{n}+$ $\left.\Lambda_{n}\right)$. Then the connected component $\mathcal{M}(\lambda)$ containing the maximal vector

$$
\begin{gathered}
M_{0}=Y_{1}(1)^{a_{1}} \cdots Y_{n}(1)^{2 a_{n}}=X_{1}(1)^{a_{1}} \cdots\left(X_{1}(n) \cdots X_{n}(1)\right)^{a_{n}} \\
\left(\text { resp. } Y_{1}(1)^{a_{1}} \cdots Y_{n}(1)^{2 a_{n}+1}=\right. \\
X_{1}(1)^{a_{1}} \cdots\left(X_{1}(n) \cdots X_{n}(1)\right)^{a_{n}} \\
\left.\times \sqrt{X_{1}(n) \cdots X_{n}(1)}\right)
\end{gathered}
$$

is characterized as the set of monomials

$$
M=X_{t_{1,1}}(1) \cdots X_{t_{1, \alpha_{1}}}(1) \cdots X_{t_{n, 1}}(n) \cdots X_{t_{n, \alpha_{n}}}(n)
$$

$\left(\right.$ resp. $\left.M=X_{t_{1,1}}(1) \cdots X_{t_{1, \alpha_{1}}}(1) \cdots X_{t_{n, 1}}(n) \cdots X_{t_{n, \alpha_{n}}}(n) \sqrt{X_{s_{1}}(1) \cdots X_{s_{n}}(n)}\right)$ satisfying the following conditions:

(i) $\alpha_{k}=a_{k}+\cdots+a_{n}$ for $k=1, \ldots, n$,

(ii) $t_{k, 1} \succeq t_{k, 2} \succeq \cdots \succeq t_{k, \alpha_{j}}$ for $k=1, \cdots, n$ (resp. $s_{k} \succeq t_{k, 1} \succeq t_{k, 2} \succeq \cdots \succeq$ $\left.t_{k, \alpha_{k}}\right)$,

(iii) for each $j=2, \ldots, n$ and $k=1, \cdots, \alpha_{j}$, 


$$
\begin{gathered}
t_{j-1, k} \succ t_{j, k} \text { or } t_{j-1, k}=t_{j, k}=0 \\
\left(\text { resp. } t_{j-1, k} \succ t_{j, k} \text { or } t_{j-1, k}=t_{j, k}=0 \text { and } s_{j-1} \succ s_{j}, \text { and } s_{j}\right. \\
(j=1, \cdots, n) \text { satisfy the condition of Proposition 2.11). }
\end{gathered}
$$

Proof. The proof is the same as that of Proposition 2.4.

Recall that any dominant integral weight $\lambda$ can be expressed as

$$
\lambda=a_{1} \omega_{1}+\cdots+a_{n} \omega_{n}+b \Lambda_{n} \quad(b=0 \text { or } 1)
$$

and a monomial $M$ in $\mathcal{M}(\lambda)$ can be expressed as

$$
M= \begin{cases}\prod_{j} X_{b_{j}}\left(n_{j}\right) & \text { if } b=0 \\ \prod_{j} X_{b_{j}}\left(n_{j}\right) \times \prod_{1 \leq k \leq n} \sqrt{X_{i_{k}}(k)} & \text { if } b=1 .\end{cases}
$$

This expression is not unique as in the $C_{n}$-case due to Lemma 2.8. Thus we will find a canonical expression for $M$ as follows. First, let $\lambda=a_{1} \omega_{1}+\cdots+a_{n} \omega_{n}$ and $M$ be a monomial in $\mathcal{M}(\lambda)$. Then $M$ can be expressed as

$$
\prod_{i \in \mathbf{B} ; j=1, \ldots, n} X_{i}(j)^{m_{i j}}
$$

Let $T(M)$ be the tableau associated with a given expression of $M$. In addition to the equivalence relations (al-1), (al-2), we will use the following equivalence relation:

(al-3) If $(0(p), 0(p))$ appears in $T(M)$, then we substitute $(n(p), \bar{n}(p))$ for $(0(p)$, $0(p))$. That is,

$$
0|0 \quad n| \bar{n}
$$

As in the $C_{n}$-case, we will denote by $[T(M)]$ the tableau obtained from $T(M)$ by applying the equivalence relations (al-1), (al-2) and (al-3), and call the corresponding monomial $[M]$ the canonical expression of $M$.

Secondly, let $\lambda=a_{1} \omega_{1}+\cdots+a_{n} \omega_{n}+\Lambda_{n}$ and let

$$
\begin{aligned}
M & =\prod_{i \in \mathbf{B} ; j=1, \cdots, n} X_{i}(j)^{m_{i j}} \times \prod_{1 \leq k \leq n} \sqrt{X_{i_{k}}(k)} \\
& =M_{p} \times \prod_{1 \leq k \leq n} \sqrt{X_{i_{k}}(k)}
\end{aligned}
$$


be a monomial in $\mathcal{M}(\lambda)$, where $M_{p} \in \mathcal{M}\left(a_{1} \omega_{1}+\cdots+a_{n} \omega_{n}\right)$. Now, we define a tableau $T(M)$ to be the tableau consisting of $T\left(\left[M_{p}\right]\right)$ and a half column located in the right-hand side of $T\left(\left[M_{p}\right]\right)$ with entries $i_{k}$ in the $k$-th row. For simplicity, we will say that there is an $\sqrt{i}(p)$ if there exists an entry $i$ lying in the $p$-th row of the half column of $T(M)$ from the bottom. In addition to the equivalence relations (al-1), (al-2) and (al-3), we will use the following equivalence relations (al-h-1)-(al-h-4) on the set of tableaux $T(M)$ : From now on, we assume that every $i(j)$ appearing in (al-h-1)-(al-h-4) lies in the rightmost column of $T\left(\left[M_{p}\right]\right)$.

(al-h-1) If $0(p), \sqrt{a}(p), \sqrt{\bar{n}}(p-1), \ldots, \sqrt{\overline{a+1}}(p-n+a)$ appear in $T(M)$, we replace them with $a(p), \sqrt{\bar{n}}(p), \ldots, \sqrt{\overline{a+1}}(p-n+a+1), \sqrt{\bar{a}}(p-n+a)$.

(al-h-2) If $\bar{a}(p), \sqrt{a}(n-a+p), \ldots, \sqrt{n}(p)$ appear in $T(M)$, we replace them with $0(p), \sqrt{a+1}(n-a+p), \ldots, \sqrt{n}(p+1) \sqrt{\bar{a}}(p)$.

(al-h-3) For $a<b$, if $\bar{a}(p), \sqrt{a}(n-a+p), \ldots, \sqrt{b-1}(n-(b-1)+p), \sqrt{b+1}(n-$ $b+p), \ldots, \sqrt{n}(1+p), \sqrt{\bar{b}}(p)$ appear in $T(M)$, we replace them with $\bar{b}(p), \sqrt{a+1}(n-a+p), \ldots, \sqrt{b}(n-(b-1)+p), \ldots, \sqrt{n}(1+p)$ and $\sqrt{\bar{a}}(p)$.

(al-h-4) For $a>b$, if $X_{a}(p), \sqrt{b}(p), \sqrt{\bar{a}}(p-(n-a+1)), \ldots, \sqrt{\overline{b+1}}(p-n+b)$ appear in $T(M)$, we replace them with $b(p), \sqrt{a}(p), \sqrt{\overline{a-1}}(p-(n-$ $a+1)), \ldots, \sqrt{b}(p-n+b)$.

That is,

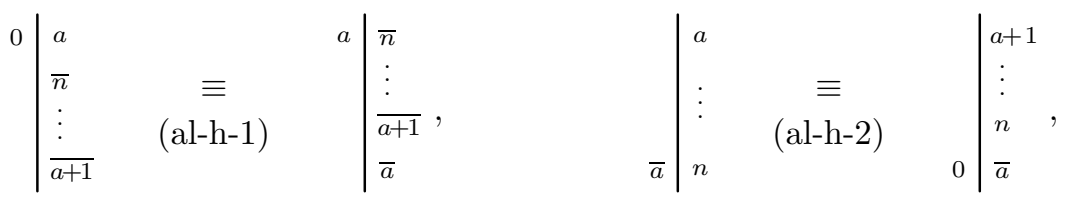

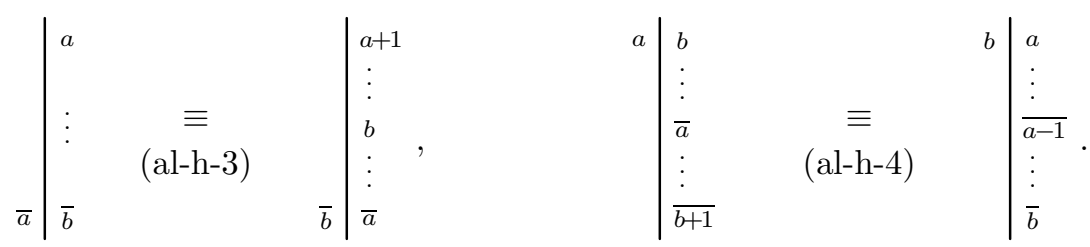

Finally, we rearrange $i(j)$ in $T(M)$, if necessary, so that the entries $i$ are weakly increasing in each row. We will also denote by $[T(M)]$ the tableau 
obtained from $T(M)$ by applying the equivalence relations (al-1)-(al-3) and (al-h-1)-(al-h-4), and call the corresponding monomial $[M]$ the canonical expression of $M$.

Example 2.14. Let $\lambda=\omega_{2}+\omega_{3}+\Lambda_{3}$ be a dominant integral weight for $B_{3}$ and let

$$
M=Y_{1}(4)^{-1} Y_{2}(2)^{2} Y_{2}(3)^{-1} Y_{3}(1) .
$$

Then $M$ can be expressed as

$$
X_{1}(3) X_{2}(2)^{2} X_{\overline{1}}(1) X_{\overline{2}}(1) \sqrt{X_{1}(3) X_{2}(2) X_{3}(1)} .
$$

At first, consider $M_{p}=X_{1}(3) X_{2}(2)^{2} X_{\overline{1}}(1) X_{\overline{2}}(1)$. Then

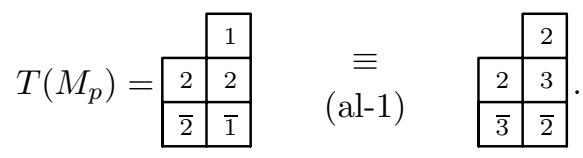

Moreover,

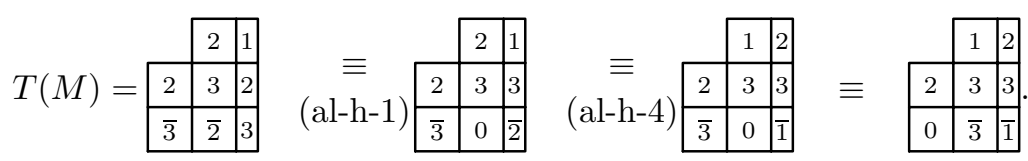

Therefore,

$$
[M]=X_{1}(3) X_{3}(2) X_{2}(2) X_{\overline{3}}(1) X_{0}(1) \sqrt{X_{2}(3) X_{3}(2) X_{\overline{1}}(1)} .
$$

From the above algorithm, we have

Theorem 2.15. Let $\lambda=a_{1} \omega_{1}+\cdots+a_{n} \omega_{n}$ (resp. $\left.a_{1} \omega_{1}+\cdots+a_{n} \omega_{n}+\Lambda_{n}\right)$. Then the connected component $\mathcal{M}(\lambda)$ containing the maximal vector $M_{0}$ given in Proposition 2.13 is characterized as the set of monomials

$$
\begin{aligned}
& M=X_{t_{1,1}}(1) \cdots X_{t_{1, \alpha_{1}}}(1) \cdots X_{t_{n, 1}}(n) \cdots X_{t_{n, \alpha_{n}}}(n) \\
& \left(\operatorname{resp} . M=X_{t_{1,1}}(1) \cdots X_{t_{1, \alpha_{1}}}(1) \cdots X_{t_{n, 1}}(n) \cdots X_{t_{n, \alpha_{n}}}(n) \sqrt{X_{s_{1}}(1) \cdots X_{s_{n}}(n)}\right)
\end{aligned}
$$

satisfying the following conditions:

(i) $\alpha_{k}=a_{k}+\cdots+a_{n}$ for $k=1, \ldots, n$,

(ii) for each $k=1, \ldots, n, t_{k, 1} \succeq t_{k, 2} \succeq \cdots \succeq t_{k, \alpha_{j}} \quad$ (resp. $s_{k} \succeq t_{k, 1} \succeq t_{k, 2}$ $\left.\succeq \cdots \succeq t_{k, \alpha_{k}}\right)$, 
(iii) for each $j=2, \ldots, n$ and $k=1, \ldots, \alpha_{j}$,

$$
\begin{gathered}
t_{j-1, k} \succ t_{j, k} \text { or } t_{j-1, k}=t_{j, k}=0 \\
\text { (resp. } t_{j-1, k} \succ t_{j, k} \text { or } t_{j-1, k}=t_{j, k}=0 \text { and } s_{j-1} \succ s_{j}, \text { and } s_{j} \\
(j=1, \cdots, n) \text { satisfy the conditions of Proposition 2.11), }
\end{gathered}
$$

(iv) there is no pair $\left(X_{t_{p, j}}(p), X_{t_{p, j+1}}(p)\right)$ with $t_{p, j}=t_{p, j+1}=0$,

(v) there is no pair $\left(X_{t_{p, k}}(p), X_{t_{q, l}}(q)\right)$ with $k \leq l, p>q$ such that

$$
t_{p, k}=a, t_{q, l}=\bar{a} \text { and } p-q=n-a,
$$

(vi) there is no pair $\left(X_{t_{p, k}}(p), X_{t_{q, l}}(q)\right)$ with $k>l, p>q$ such that

$$
t_{p, k}=a, t_{q, l}=\bar{a} \text { and } p-q=n-a+1 .
$$

\section{$\S 2.3$. The $U_{q}\left(D_{n}\right)$-crystal $\mathcal{M}(\lambda)$}

Let $U_{q}\left(\mathfrak{s o}_{2 n}\right)$ be the quantum special orthogonal algebra. Then for $i \in I$ and $m \in \mathbf{Z}$, we have

$$
A_{i}(m)= \begin{cases}Y_{n-3}(m+1)^{-1} Y_{n-2}(m) Y_{n-2}(m+1) Y_{n-1}(m)^{-1} Y_{n}(m)^{-1} \\ Y_{n-2}(m+1)^{-1} Y_{n-1}(m) Y_{n-1}(m+1) & \text { for } i=n-2, \\ Y_{n-2}(m+1)^{-1} Y_{n}(m) Y_{n}(m+1) & \text { for } i=n-1, \\ Y_{i-1}(m+1)^{-1} Y_{i}(m) Y_{i}(m+1) Y_{i+1}(m)^{-1} & \text { for } i=n,\end{cases}
$$

Set

$$
\omega_{i}= \begin{cases}\lambda_{i} & \text { for } i=1, \cdots, n-2, \\ \lambda_{n-1}+\lambda_{n} & \text { for } i=n-1 \\ 2 \lambda_{n} & \text { for } i=n \\ 2 \lambda_{n-1} & \text { for } i=n+1\end{cases}
$$

Then every dominant integral weight $\lambda$ can be expressed as one of the following:
(i) $a_{1} \omega_{1}+\cdots+a_{n} \omega_{n}$
(ii) $a_{1} \omega_{1}+\cdots+a_{n-1} \omega_{n-1}+a_{n+1} \omega_{n+1}$,
(iii) $a_{1} \omega_{1}+\cdots+a_{n} \omega_{n}+\Lambda_{n}$,
(iv) $a_{1} \omega_{1}+\cdots+a_{n-1} \omega_{n-1}+a_{n+1} \omega_{n+1}+\Lambda_{n-1}$, 
where $a_{i} \in \mathbf{Z}_{\geq 0}$ for all $i=1, \cdots, n+1$.

We first consider the case when $\lambda=\Lambda_{k}$. Let $M_{0}=Y_{k}(m)$ for $m \in \mathbf{Z}$. Then we know that the connected component containing $M_{0}$ is isomorphic to $B\left(\Lambda_{k}\right)$ over $U_{q}(\mathfrak{g})$. For simplicity, we will take $M_{0}=Y_{k}(1)$.

Let $\mathbf{B}=\{1,2, \ldots, n, \overline{1} \cdots \bar{n}\}$ and define an ordering on $\mathbf{B}$ by

$$
1 \prec 2 \prec \cdots \prec n-1 \prec n, \bar{n} \prec \overline{n-1} \prec \cdots \prec \overline{1} .
$$

For $m \in \mathbf{Z}$, we introduce new variables

$$
\begin{aligned}
& X_{i}(m)= \begin{cases}Y_{i-1}(m+1)^{-1} Y_{i}(m) & \text { for } i=1, \ldots, n-2, n, \\
Y_{n-2}(m+1)^{-1} Y_{n-1}(m) Y_{n}(m) & \text { for } i=n-1,\end{cases} \\
& X_{\bar{i}}(m)= \begin{cases}Y_{i-1}(m+(n-i)) Y_{i}(m+(n-i))^{-1} & \text { for } i=1, \ldots, n-2, \\
Y_{n-2}(m+1) Y_{n-1}(m+1)^{-1} Y_{n}(m+1)^{-1} & \text { for } i=n-1, \\
Y_{n-1}(m) Y_{n}(m+1)^{-1} & \text { for } i=n .\end{cases}
\end{aligned}
$$

Also, for a monomial $M=\sqrt{Y_{i}(k) Y_{i}(k+1)}$, we denote by $|M|$ the monomial $Y_{i}(k)$, which has the same weight as that of $M$.

Lemma 2.16. For $a, b, p, q=1, \ldots, n$, and $i=1, \ldots, n-2$, we have

$$
\begin{aligned}
& X_{i}(p) X_{\bar{i}}(q)=X_{i+1}(p) X_{\overline{i+1}}(q) \quad \text { if } p-q=(n-1)-i \\
& X_{n}(p) X_{\bar{n}}(p)=X_{n-1}(p) X_{\overline{n-1}}(p), \\
& X_{\bar{a}}(0)\left|\sqrt{X_{1}(n-1) \cdots X_{n}(0)}\right| \\
& =\left\{\begin{array}{cc}
X_{n}(0) \mid \sqrt{X_{1}(n-1) \cdots X_{a-1}(n-a+1)} \quad(a \neq n), \\
\quad \times \sqrt{X_{a+1}(n-a) \cdots X_{\bar{n}}(1) X_{\bar{a}}(0)} \mid \\
X_{n-1}(0)\left|\sqrt{X_{1}(n-1) \cdots X_{n-2}(2) X_{\bar{n}}(1) X_{\overline{n-1}}(0)}\right|(a=n),
\end{array}\right. \\
& X_{\bar{a}}(p) \mid \sqrt{X_{a}(n-a+p) \cdots X_{b-1}(n-b+1+p)} \\
& \quad \times \sqrt{X_{b+1}(n-b+p) \cdots X_{n}(1+p) X_{\bar{b}}(p)} \mid \\
& =X_{\bar{b}}(p)\left|\sqrt{X_{a+1}(n-a+p) \cdots X_{n}(1+p) X_{\bar{a}}(p)}\right| \text { for } \quad a<b, \\
& X_{a}(p)\left|\sqrt{X_{b}(p) X_{\bar{a}}(p-(n-a+1)) \cdots X_{\overline{b+1}}(p-n+b)}\right| \\
& =X_{b}(p)\left|\sqrt{X_{a}(p) X_{a-1}(p-(n-a+1)) \cdots X_{\bar{b}}(p-n+b)}\right| \\
& \quad \text { for } a>b .
\end{aligned}
$$


Proposition 2.17. For $k=1, \cdots, n-1$, let $M_{0}=Y_{k}(1)$ be a maximal vector of weight $\omega_{k}$. Then the connected component $\mathcal{M}\left(\omega_{k}\right)$ of $\mathcal{M}$ containing $M_{0}$ is characterized as

$$
\begin{aligned}
& \mathcal{M}\left(\omega_{k}\right)= \\
& \quad\left\{X_{i_{1}}(1) X_{i_{2}}(2) \cdots X_{i_{k}}(k) \mid i_{j} \succ i_{j+1} \text { or }\left(i_{j}, i_{j+1}\right)=(n, \bar{n}) \text { or }(\bar{n}, n) .\right\} .
\end{aligned}
$$

Proof. The proof is similar to that of Proposition 2.1.

By Lemma 2.16, we have

Proposition 2.18. For $k=1, \ldots, n-1$, let $M_{0}=Y_{k}(1)$ be a maximal vector of weight $\omega_{k}$. Then the connected component $\mathcal{M}\left(\omega_{k}\right)$ of $\mathcal{M}$ containing $M_{0}$ is characterized as

$$
\begin{aligned}
& \mathcal{M}\left(\omega_{k}\right)= \\
& \left\{\begin{array}{c}
\text { (i) } i_{j} \succ i_{j+1} \text { or }\left(i_{j}, i_{j+1}\right)=(n, \bar{n}) \text { or }(\bar{n}, n), \\
X_{i_{1}}(1) X_{i_{2}}(2) \cdots X_{i_{k}}(k) \mid \text { (ii) there is no } p, q \text { such that } i_{p}=a, i_{q}=\bar{a} \\
\text { and } p-q=(n-1)-a .
\end{array}\right.
\end{aligned}
$$

Proposition 2.19. Let $M_{0}=Y_{n}(1) Y_{n}(2)$ (resp. $\left.Y_{n-1}(1) Y_{n-1}(2)\right)$ be a maximal vector of weight $\omega_{n}=2 \Lambda_{n}$ (resp. $\left.\omega_{n+1}=2 \Lambda_{n-1}\right)$. Then the connected component $\mathcal{M}\left(\omega_{n}\right)$ (resp. $\left.\mathcal{M}\left(\omega_{n+1}\right)\right)$ of $\mathcal{M}$ containing $M_{0}$ is characterized as the set of monomials of the form

$$
X_{i_{1}}(1) X_{i_{2}}(2) \cdots X_{i_{n}}(n)
$$

satisfying the following conditions:

(i) $i_{j} \succ i_{j+1}$ or $\left(i_{j}, i_{j+1}\right)=(n, \bar{n})$ or $(\bar{n}, n)$,

(ii) $i_{k}=n$ implies $k$ is even (resp. odd) and $i_{k}=\bar{n}$ implies $k$ is odd (resp. even),

(iii) $i_{p}=a$ and $i_{q}=\bar{a}$ implies that $p-q$ is not $n-a$.

Proof. Since the proof for $\mathcal{M}\left(\omega_{n+1}\right)$ is the same as that for $\mathcal{M}\left(\omega_{n}\right)$, we focus on $\mathcal{M}\left(\omega_{n}\right)$. As in Proposition 2.1, it suffices to prove (C1) and (C2). It is easy to see that (C2) is satisfied.

To prove $(\mathbf{C} 1)$, suppose $\tilde{f}_{i} M \neq 0$. By the same argument in Proposition 2.1, we see that $\tilde{f}_{i} M$ satisfies (i).

Now, assume that $\tilde{f}_{i} M$ does not satisfy the condition (ii). Then there are the following two possibilities: 
(ii-a) There exists $k$ such that $n-k$ is odd and $i_{k}=n-1$ is changed to $n$ by $\tilde{f}_{n-1}$.

(ii-b) There exists $k$ such that $n-k$ is even and $i_{k}=n-1$ is changed to $\bar{n}$ by $\tilde{f}_{n}$.

Note that for any monomial $M \in \mathcal{M}\left(\omega_{n}\right)$, there is $X_{n}(\cdot)$ or $X_{\bar{n}}(\cdot)$ in $M$. Moreover, if the condition (ii) is satisfied for some $k$ such that $i_{k}=n$ or $\bar{n}$, then it is satisfied for all $k$ since $X_{n}(\cdot)$ and $X_{\bar{n}}(\cdot)$ appear in $M$ alternately and successively. Therefore, the above two possibilities cannot occur and hence $\tilde{f}_{i} M$ satisfies the condition (ii).

Secondly, assume that $\tilde{f}_{i} M$ does not satisfy the condition (iii). Then there are the following two possibilities:

(iii-a) There exists a pair $(p, q)$ such that $p-q=n-a, i_{q}=\bar{a}$ and $i_{p}=a-1$ is changed to $a$ by $\tilde{f}_{a-1}$.

(iii-b) There exists a pair $(p, q)$ such that $p-q=n-a, i_{p}=a$ and $i_{q}=\overline{a+1}$ is changed to $\bar{a}$ by $\tilde{f}_{a}$.

Consider the case (iii-a). Note that $X_{\overline{a-1}}(n-q-1)$ cannot appear in $M$ because $q+1-p=n-(a-1)$. Moreover, since the cardinality of the set $\left\{i_{1}, \ldots, i_{p-1}, i_{q+1}, \ldots, i_{n}\right\}$ is $a-1$, there is a pair $(r, s)$ such that $i_{r}=b, i_{s}=\bar{b}$ and $r-s=n-b$, which is a contradiction.

In case (iii-b), since $p-q=n-a$, we have

$$
\begin{aligned}
X_{a}(p) & =Y_{a-1}(p+1)^{-1} Y_{a}(p), \\
X_{\overline{a+1}}(q) & =Y_{a}(q+n-a-1) Y_{a+1}(q+n-a-1)^{-1} \\
& =Y_{a}(p-1) Y_{a+1}(p-1)^{-1} .
\end{aligned}
$$

Therefore, $\tilde{f}_{a} M$ is obtained from $M$ by multiplying $A_{a}(p)^{-1}=X_{a}(p)^{-1} X_{a+1}(p)$, which contradicts to (ii). Therefore, $\tilde{f}_{i} M \in \mathcal{M}\left(\omega_{n}\right) \cup\{0\}$.

Similarly, one can prove $\tilde{e}_{i} M \in \mathcal{M}\left(\omega_{n}\right) \cup\{0\}$.

Combining Lemma 2.16 and Proposition 2.19, we obtain

Proposition 2.20. Let $M_{0}=Y_{n}(1) Y_{n}(2)$ (resp. $\left.Y_{n-1}(1) Y_{n-1}(2)\right)$ be a maximal vector of weight $\omega_{n}=2 \Lambda_{n}$ (resp. $\left.\omega_{n+1}=2 \Lambda_{n-1}\right)$. Then the connected component $\mathcal{M}\left(\omega_{n}\right)$ (resp. $\left.\mathcal{M}\left(\omega_{n+1}\right)\right)$ of $\mathcal{M}$ containing $M_{0}$ is characterized as the set of monomials of the form

$$
X_{i_{1}}(1) X_{i_{2}}(2) \cdots X_{i_{n}}(n)
$$


satisfying the following conditions:

(i) $i_{j} \succ i_{j+1}$ or $\left(i_{j}, i_{j+1}\right)=(n, \bar{n})$ or $(\bar{n}, n)$,

(ii) $i_{k}=n$ implies $k$ is even (resp. odd) and $i_{k}=\bar{n}$ implies $k$ is odd (resp. even),

(iii) $i_{p}=a$ and $i_{q}=\bar{a}$ implies that $p-q$ is neither $n-a$ nor $(n-1)-a$.

Proposition 2.21. Let $M_{0}=Y_{n}(1)$ (resp. $\left.Y_{n-1}(1)\right)$ be a maximal vector of weight $\Lambda_{n}$ (resp. $\left.\Lambda_{n-1}\right)$. Then the connected component $\mathcal{M}\left(\Lambda_{n}\right)$ (resp. $\left.\mathcal{M}\left(\Lambda_{n-1}\right)\right)$ of $\mathcal{M}$ containing $M_{0}$ is characterized as the set of monomials of the form

$$
\left|\sqrt{X_{i_{1}}(1) X_{i_{2}}(2) \cdots X_{i_{n}}(n)}\right|
$$

satisfying the following conditions:

(i) $1 \preceq i_{1} \prec i_{2} \prec \cdots \prec i_{n} \preceq \overline{1}$,

(ii) there is no pair $\left(i_{p}=a, i_{q}=\bar{a}\right)$ for all $p, q=1, \ldots, n$,

(iii) $i_{k}=n$ implies $k$ is even (resp. odd),

(iv) $i_{k}=\bar{n}$ implies $k$ is odd (resp. even).

Proof. The proof is similar to that of Proposition 2.11.

Remark 2.22. As in Remark 2.12, we can see that $\left|\sqrt{X_{i_{1}}(1) X_{i_{2}}(2) \cdots X_{i_{n}}(n)}\right|$ satisfying (i)-(iv) is a monomial in $Y_{i}(k)$ 's.

Now, we consider the general case.

\section{Proposition 2.23.}

(a) Let $\lambda=a_{1} \omega_{1}+\cdots+a_{n} \omega_{n}$ (resp. $\left.a_{1} \omega_{1}+\cdots+a_{n-1} \omega_{n-1}+a_{n+1} \omega_{n+1}\right)$. Then the connected component $\mathcal{M}(\lambda)$ containing the maximal vector

$$
\begin{aligned}
M_{0} & =Y_{1}(1)^{a_{1}} \cdots\left(Y_{n-1}(1) Y_{n}(1)\right)^{a_{n-1}}\left(Y_{n}(1) Y_{n}(2)\right)^{a_{n}} \\
& =X_{1}(1)^{a_{1}} \cdots\left(X_{1}(n) \cdots X_{n}(1)\right)^{a_{n}} \\
\text { resp. } M_{0} & =Y_{1}(1)^{a_{1}} \cdots\left(Y_{n-1}(1) Y_{n}(1)\right)^{a_{n-1}}\left(Y_{n-1}(1) Y_{n-1}(2)\right)^{a_{n+1}} \\
& \left.=X_{1}(1)^{a_{1}} \cdots\left(X_{1}(n) \cdots X_{\bar{n}}(1)\right)^{a_{n+1}}\right)
\end{aligned}
$$

is characterized as the set of monomials

$$
M=X_{t_{1,1}}(1) \cdots X_{t_{1, \alpha_{1}}}(1) \cdots X_{t_{n, 1}}(n) \cdots X_{t_{n, \alpha_{n}}}(n)
$$

satisfying the following conditions: 
(i) $\alpha_{k}=a_{k}+\cdots+a_{n}$ for $k=1, \cdots, n$,

(ii) for each $k=1, \cdots, n, t_{k, 1} \succeq t_{k, 2} \succeq \cdots \succeq t_{k, \alpha_{k}}$,

(iii) for each $j=2, \ldots, n$ and $k=1, \ldots, \alpha_{j}$,

$$
t_{j-1, k} \succ t_{j, k}, \text { or }\left(t_{j-1, k}, t_{j, k}\right)=(n, \bar{n}) \text { or }(\bar{n}, n),
$$

(iv) for each $j$, if every $t_{i, j}(i=1, \ldots, n)$ appears in $M$, then $t_{i, j}(j=1, \ldots, n)$ satisfy the condition of Proposition 2.19.

(b) Let $\lambda=a_{1} \omega_{1}+\cdots+a_{n} \omega_{n}+\Lambda_{n}$ (resp. $a_{1} \omega_{1}+\cdots+a_{n-1} \omega_{n-1}+a_{n+1} \omega_{n+1}+$ $\left.\Lambda_{n-1}\right)$. Then the connected component $\mathcal{M}(\lambda)$ containing the maximal vector

$$
\begin{aligned}
M_{0} & =Y_{1}(1)^{a_{1}} \cdots\left(Y_{n-1}(1) Y_{n}(1)\right)^{a_{n-1}}\left(Y_{n}(1) Y_{n}(2)\right)^{a_{n}} Y_{n}(1) \\
& =X_{1}(1)^{a_{1}} \cdots\left(X_{1}(n) \cdots X_{n}(1)\right)^{a_{n}}\left|\sqrt{X_{1}(n) \cdots X_{n}(1)}\right| \\
\left(\operatorname{resp} . M_{0}\right. & =Y_{1}(1)^{a_{1}} \cdots\left(Y_{n-1}(1) Y_{n}(1)\right)^{a_{n-1}}\left(Y_{n-1}(1) Y_{n-1}(2)\right)^{a_{n+1}} Y_{n-1}(1) \\
& \left.=X_{1}(1)^{a_{1}} \cdots\left(X_{1}(n) \cdots X_{\bar{n}}(1)\right)^{a_{n+1}}\left|\sqrt{X_{1}(n) \cdots X_{\bar{n}}(1)}\right|\right)
\end{aligned}
$$

is characterized as the set of monomials

$$
M=X_{t_{1,1}}(1) \cdots X_{t_{1, \alpha_{1}}}(1) \cdots X_{t_{n, 1}}(n) \cdots X_{t_{n, \alpha_{n}}}(n)\left|\sqrt{X_{s_{1}}(1) \cdots X_{s_{n}}(n)}\right|
$$

satisfying the following conditions:

(i) $\alpha_{k}=a_{k}+\cdots+a_{n}$ for $k=1, \ldots, n$,

(ii) for each $k=1, \ldots, n, s_{k} \geq t_{k, 1} \succeq t_{k, 2} \succeq \cdots \succeq t_{k, \alpha_{k}}$,

(iii) for each $i=1, \ldots, M_{j}$ and $j=1, \ldots, n$,

$$
t_{j-1, k} \succ t_{j, k}, \text { or }\left(t_{j-1, k}, t_{j, k}\right)=(n, \bar{n}) \text { or }(\bar{n}, n), s_{j} \prec s_{j-1},
$$

(iv) for each $j$, if every $t_{i, j}(i=1, \ldots, n)$ appears in $M$, then $t_{i, j}(i=1, \ldots, n)$ satisfy the conditions of Proposition 2.19,

(v) $s_{j}(j=1, \ldots, n)$ satisfy the conditions of Proposition 2.21. 
Proof. It can be proved by the same argument in Proposition 2.4.

Let $\lambda$ be a dominant integral weight. Then $\lambda$ can be expressed as one of (2.16). Let $M$ be a monomial in $\mathcal{M}(\lambda)$, which can be expressed as

$$
M= \begin{cases}\prod_{j} X_{b_{j}}\left(n_{j}\right) & \text { if } \lambda \text { is type of (i) or (ii) } \\ \prod_{j} X_{b_{j}}\left(n_{j}\right) \times\left|\prod_{1 \leq k \leq n} \sqrt{X_{i_{k}}(k)}\right| & \text { if } \lambda \text { is type of (iii) or (iv) } .\end{cases}
$$

Since this expression is not unique, we will choose a canonical expression of $M$.

First, let $\lambda=a_{1} \omega_{1}+\cdots+a_{n} \omega_{n}$ or $a_{1} \omega_{1}+\cdots+a_{n-1} \omega_{n-1}+a_{n+1} \omega_{n+1}$ and $M$ be a monomial in $\mathcal{M}(\lambda)$. As in the other cases, given an expression of $M$, we can associate a tableau $T(M)$. In addition to the equivalence relations (al-1), (al-2) given in $C_{n-1}$-case, we will use the following equivalence relation:

(al-3) If there exist $n(p)$ and $\bar{n}(p)$ in $T(M)$, then we substitute $n-1(p)$ and $\overline{n-1}(p)$ for $n(p)$ and $\bar{n}(p)$. That is,

$$
\bar{n}|n \equiv n| \bar{n} \equiv n-1 \mid \overline{n-1}
$$

We will denote by $[T(M)]$ the tableau obtained from $T(M)$ by applying the equivalence relations (al-1), (al-2) and (al-3), and call the corresponding monomial $[M]$ the canonical expression of $M$.

Secondly, let $\lambda=a_{1} \omega_{1}+\cdots+a_{n} \omega_{n}+\Lambda_{n}$ or $a_{1} \omega_{1}+\cdots+a_{n-1} \omega_{n-1}+$ $a_{n+1} \omega_{n+1}+\Lambda_{n-1}$ and

$$
\begin{aligned}
M & =\prod_{i \in \mathbf{B} ; j=1, \cdots, n} X_{i}(j)^{m_{i j}} \times \prod_{1 \leq k \leq n} \sqrt{X_{i_{k}}(k)} \\
& =M_{p} \times \prod_{1 \leq k \leq n} \sqrt{X_{i_{k}}(k)}
\end{aligned}
$$

be a monomial in $\mathcal{M}(\lambda)$, where $M_{p} \in \mathcal{M}\left(a_{1} \omega_{1}+\cdots+a_{n} \omega_{n}\right)$ or $\mathcal{M}\left(a_{1} \omega_{1}+\right.$ $\left.\cdots+a_{n-1} \omega_{n-1}+a_{n+1} \omega_{n+1}\right)$. Now, we define a tableau $T(M)$ to be the tableau consisting of $T\left(\left[M_{p}\right]\right)$ and a half column located in the right hand side of $T\left(\left[M_{p}\right]\right)$ with entries $i_{k}$ in the $k$-th row. In addition to the equivalence relations (al-1), (al-2) and (al-3), we will use the following equivalence relations (al-h-1)-(alh-4) on the set of tableaux $T(M)$ : From now on, every $i(j)$ appeared in (al$\mathrm{h}-1)-(\mathrm{al}-\mathrm{h}-4)$ lies in the rightmost column of $T\left(\left[M_{p}\right]\right)$. Moreover, (al-h-4) is applied only when the length of the rightmost column of $T\left(\left[M_{p}\right]\right)$ is not $n$. 
(al-h-1) If there are $\bar{a}(1), \sqrt{1}(n), \ldots, \sqrt{n}(1)$, we replace them with $n(1), \sqrt{1}(n)$, $\ldots, \sqrt{a-1}(n-a+2), \sqrt{a+1}(n-a+1), \ldots, \sqrt{\bar{n}}(2), \sqrt{\bar{a}}(1)$ for $a \neq n$ and $n-1(1), \sqrt{1}(n), \ldots, \sqrt{n-2}(3), \sqrt{\bar{n}}(2), \sqrt{\overline{n-1}}(1)$ for $a=n$.

(al-h-2) If there are $\bar{a}(p), \sqrt{a}(n-a+p), \ldots, \sqrt{b-1}(n-(b-1)+p), \sqrt{b+1}(n-b+$ $p), \ldots, \sqrt{n}(1+p), \sqrt{\bar{b}}(p)$, we replace them with $\bar{b}(p), \sqrt{a+1}(n-a+p)$, $\ldots, \sqrt{b}(n-(b-1)+p), \ldots, \sqrt{n}(1+p)$ and $\sqrt{\bar{a}}(p)$.

(al-h-3) If there are $a(p), \sqrt{b}(p), \sqrt{\bar{a}}(p-(n-a+1)), \ldots, \sqrt{\overline{b+1}}(p-n+b)$, we replace them with $b(p), \sqrt{a}(p), \sqrt{a-1}(p-(n-a+1)), \ldots, \sqrt{b}(p-n+b)$.

(al-h-4) Assume that there are $a(p)$ and $\sqrt{\bar{a}}(q)$ such that $p-q=n-a$. Let $b$ be the largest element in $I$ such that $\sqrt{b}(r)(r>p)$ exists. Then $a(p)$, $\sqrt{\bar{a}}(q)$ and $\sqrt{b}(r)$ are changed to $b(p), \sqrt{\bar{b}}(q)$ and $\sqrt{a}(r)$.

That is,

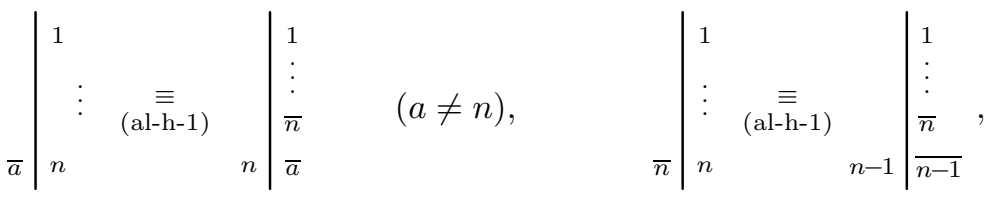

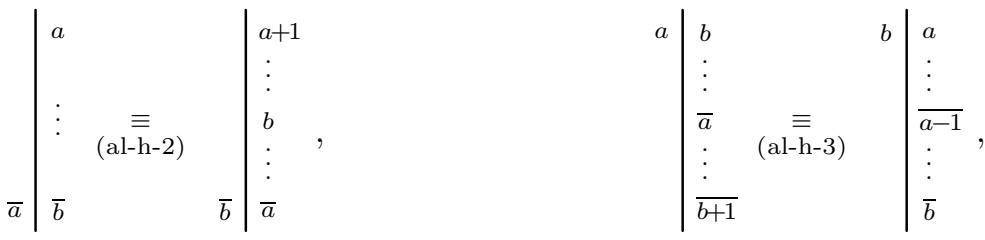

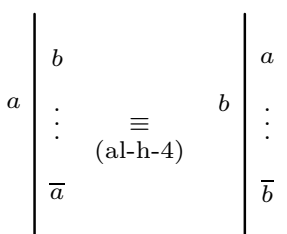

Finally, we rearrange $i(j)$ in $T(M)$, if necessary, so that the entries $i$ are weakly increasing in each row. We will denote by $[T(M)]$ the tableau obtained from $T(M)$ by applying the equivalence relations (al-1)-(al-3) and (al-h-1)(al-h-4), and call the corresponding monomial $[M]$ the canonical expression of M. 
Example 2.24. Let $\lambda=\omega_{2}+\omega_{4}+\Lambda_{4}$ for $D_{4}$ and let

$$
M=Y_{1}(3) Y_{1}(4) Y_{2}(2) Y_{2}(3)^{-1} Y_{3}(3)^{-1} Y_{3}(4)^{-1} Y_{4}(1) .
$$

Then $M$ can be expressed as

$$
X_{1}(4) X_{4}(3) X_{\overline{3}}(2) X_{3}(2) X_{\overline{2}}(1) X_{\overline{3}}(1)\left|\sqrt{X_{1}(4) X_{2}(3) X_{3}(2) X_{4}(1)}\right| .
$$

Moreover,

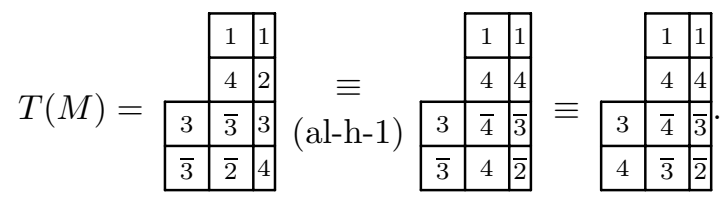

Therefore, we have

$$
[M]=X_{1}(4) X_{4}(3) X_{\overline{4}}(2) X_{3}(2) X_{\overline{3}}(1) X_{4}(1)\left|\sqrt{X_{1}(4) X_{4}(3) X_{\overline{3}}(2) X_{\overline{2}}(1)}\right| .
$$

By the above algorithm, we have

\section{Theorem 2.25.}

(a) Let $\lambda=a_{1} \omega_{1}+\cdots+a_{n} \omega_{n}$ (resp. $\left.a_{1} \omega_{1}+\cdots+a_{n-1} \omega_{n-1}+a_{n+1} \omega_{n+1}\right)$. Then the connected component $\mathcal{M}(\lambda)$ containing the maximal vector $M_{0}$ given in Proposition 2.23 (a) is characterized as the set of monomials

$$
M=X_{t_{1,1}}(1) \cdots X_{t_{1, \alpha_{1}}}(1) \cdots X_{t_{n, 1}}(n) \cdots X_{t_{n, \alpha_{n}}}(n)
$$

satisfying the following conditions:

(i) $\alpha_{k}=a_{k}+\cdots+a_{n}$ for $k=1, \ldots, n$,

(ii) for each $k=1, \ldots, n, t_{k, 1} \succeq t_{k, 2} \succeq \cdots \succeq t_{k, \alpha_{k}}$,

(iii) for each $j=2, \ldots, n$ and $k=1, \ldots, \alpha_{j}$,

$$
t_{j-1, k} \succ t_{j, k}, \text { or }\left(t_{j-1, k}, t_{j, k}\right)=(n, \bar{n}) \text { or }(\bar{n}, n),
$$

(iv) there is no pair $\left(X_{t_{p, k}}(p), X_{t_{q, l}}(q)\right)$ with $k \leq l, p>q$ such that

$$
t_{p, k}=a, t_{q, l}=\bar{a} \text { and } p-q=(n-1)-a,
$$

(v) there is no pair $\left(X_{t_{p, k}}(p), X_{t_{q, l}}(q)\right)$ with $k>l, p>q$ such that 


$$
t_{p, k}=a, t_{q, l}=\bar{a} \text { and } p-q=n-a,
$$

(vi) for each $j$, if every $t_{i, j}(i=1, \ldots, n)$ exists in $M$, then $t_{i, j}(j=1, \ldots, n)$ satisfy the conditions of Proposition 2.19.

(b) Let $\lambda=a_{1} \omega_{1}+\cdots+a_{n} \omega_{n}+\Lambda_{n}$ (resp. $a_{1} \omega_{1}+\cdots+a_{n-1} \omega_{n-1}+a_{n+1} \omega_{n+1}+$ $\left.\Lambda_{n-1}\right)$. Then the connected component $\mathcal{M}(\lambda)$ containing the maximal vector $M_{0}$ given in Proposition 2.23 (b) is characterized as the set of monomials

$$
M=X_{t_{1,1}}(1) \cdots X_{t_{1, \alpha_{1}}}(1) \cdots X_{t_{n, 1}}(n) \cdots X_{t_{n, \alpha_{n}}}(n)\left|\sqrt{X_{s_{1}}(1) \cdots X_{s_{n}}(n)}\right|
$$

satisfying the following conditions:

(i) $\alpha_{k}=a_{k}+\cdots+a_{n}$ for $k=1, \ldots, n$,

(ii) for each $k=1, \ldots, n, s_{k} \succeq t_{k, 1} \succeq t_{k, 2} \succeq \cdots \succeq t_{k, \alpha_{k}}$,

(iii) for each $j=2, \ldots, n$ and $k=1, \ldots, \alpha_{j}$,

$$
t_{j-1, k} \succ t_{j, k}, \text { or }\left(t_{j-1, k}, t_{j, k}\right)=(n, \bar{n}) \text { or }(\bar{n}, n),
$$

(iv) there is no pair $\left(X_{t_{p, k}}(p), X_{t_{q, l}}(q)\right)$ with $k \leq l, p>q$ such that

$$
t_{p, k}=a, t_{q, l}=\bar{a} \text { and } p-q=(n-1)-a,
$$

(v) there is no pair $\left(X_{t_{p, k}}(p), X_{t_{q, l}}(q)\right)$ with $k>l, p>q$ such that

$$
t_{p, k}=a, t_{q, l}=\bar{a} \text { and } p-q=n-a,
$$

(vi) for each $j$, if every $t_{i, j}(i=1, \cdots, n)$ exists in $M$, then $t_{i, j}(j=1, \cdots, n)$ satisfy the condition of Proposition 2.19,

(vii) $s_{j}(j=1, \cdots, n)$ satisfy the conditions of Proposition 2.21 .

We close this section with a remark, which reveals the intrinsic property of monomial realization.

Remark 2.26. Let $\lambda, \mu$ and $\tau$ be the dominant integral weights such that $\lambda+\mu=\tau$ for the classical Lie algebras $\mathfrak{g}=A_{n}, C_{n}, B_{n}$ and $D_{n}$. Then $\mathcal{M}(\tau)$ is the set of products of the monomials in $\mathcal{M}(\lambda)$ and $\mathcal{M}(\mu)$. That is,

$$
\mathcal{M}(\tau)=\left\{M M^{\prime} \mid M \in \mathcal{M}(\lambda), \quad M^{\prime} \in \mathcal{M}(\mu)\right\} .
$$




\section{$\S 3 . \quad$ The Connection with Young Tableaux}

In this section, we will give explicit bijections between the monomial realization, reverse Young tableau realization and the Kashiwara-Nakashima tableau realization. In [10], Kim and Shin gave an explicit crystal isomorphism between the reverse Young tableau realization and the Kashiwara-Nakashima tableau realization using the bumping procedure. Thus, we will focus on the connection between Nakajima's monomials and the reverse Young tableaux.

For a sequence of half integers $\lambda_{j} \in \frac{1}{2} \mathbf{Z}(j=1, \cdots, n)$ such that $\lambda_{j}-\lambda_{j+1} \in$ $\mathbf{Z}_{\geq 0}$, we associate a diagram $Y=\left(\lambda_{1}, \cdots, \lambda_{n}\right)$ with $n$ rows whose $j$-th row (from the bottom) has length $\left|\lambda_{j}\right|$.

Definition 3.1. Let $Y=\left(\lambda_{1}, \cdots, \lambda_{n}\right)$ be a diagram such that $\lambda_{j} \in \frac{1}{2} \mathbf{Z}$ $(j=1, \cdots, n)$ and $\lambda_{j}-\lambda_{j+1} \in \mathbf{Z}_{\geq 0}(j=1, \cdots, n-1)$.

(a) $Y$ is called generalized reverse Young diagram of type $C_{n}$ (resp. $B_{n}$ ) if $\lambda_{j} \in \mathbf{Z}_{\geq 0}$ (resp. $\left.\lambda_{j} \in \frac{1}{2} \mathbf{Z}_{\geq 0}\right)$ for all $j$.

(b) $Y$ is called generalized reverse Young diagram of type $D_{n}$ if $\lambda_{j} \in \frac{1}{2} \mathbf{Z}_{\geq 0}$ for all $j=1, \cdots, n-1$ and $\lambda_{n-1} \geq\left|\lambda_{n}\right|$.

In [10], in an attempt to understand the connection between the Young wall realization and the Kashiwara-Nakashima tableau realization, Kim and Shin gave a new realization $S(\lambda)$ of crystal basis for the classical Lie algebras using the tableaux which are fillings of a given generalized reverse Young diagram. The entries of the tableaux in $S(\lambda)$ satisfy the same conditions for the Kashiwara-Nakashima tableaux. We refer the readers to [10] for more details.

\section{$\S 3.1 . \quad U_{q}\left(C_{n}\right)$ type}

Proposition 3.2. For $k=1, \cdots, n$, there is a crystal isomorphism $\psi$ : $\mathcal{M}\left(\Lambda_{k}\right) \rightarrow S\left(\Lambda_{k}\right)$.

Proof. Let $M=X_{i_{1}}(1) \cdots X_{i_{k}}(k)$ be a monomial in $\mathcal{M}\left(\Lambda_{k}\right)$. If there is no pair $\left(X_{a}(p), X_{\bar{a}}(q)\right)$ such that $p-q<n-a$, we define $\psi(M)$ to be the tableau of one column with entries $i_{1}, \cdots, i_{k}$ from bottom to top.

Suppose that there is a pair $\left(X_{a}(p), X_{\bar{a}}(q)\right)$ such that $p-q<n-a$. Then we define $\psi(M)$ to be the tableau of one column with entries $i_{1}, \cdots, \overline{a+1}, \cdots, a+$ $1, \cdots, i_{k}$ from bottom to top, where $\overline{a+1}$ and $a+1$ lie at the $q$-th and $p$-th rows, respectively. If there are several pairs $\left(X_{a}(p), X_{\bar{a}}(q)\right)$ such that $p-q<n-a$ 
( $a=1, \cdots, n-1)$, then $\psi(M)$ is defined by applying the above rule repeatedly from $a=1$ to $n-1$.

By the definition of $[M]$, we may assume that $[M]$ is obtained from an expression of $M$ by replacing one pair $\left(X_{a}(p), X_{\bar{a}}(q)\right)$ such that $p-q=n-a$ with $\left(X_{a+1}(p), X_{\overline{a+1}}(q)\right)$. It follows that

$$
(k-(p+1)+1)+(q+1) \leq n-(p-q)+1=a+1 .
$$

Therefore, for the monomial $[M]$ with no pair $\left(X_{a}(p), X_{\bar{a}}(q)\right)$ such that $p-q<$ $n-a, \psi(M)$ satisfies the condition for the tableaux in $S\left(\Lambda_{k}\right)$.

Consider the case that there is a pair $\left(X_{a}(p), X_{\bar{a}}(q)\right)$ such that $p-q<n-a$ in $[M]$. We may assume that $[M]$ has only one pair $\left(X_{a}(p), X_{\bar{a}}(q)\right)$ such that $p-q<n-a$. If there is no pair $\left(X_{b}(r), X_{\bar{b}}(s)\right)$ with $b<a$, then

$$
(k-(p+1)+1)+(q+1) \leq a+1 .
$$

Suppose that there is a pair $\left(X_{b}(r), X_{\bar{b}}(s)\right)$ with $b<a$. Let $b$ be the largest index such that $\left(X_{b}(r), X_{\bar{b}}(s)\right)$ appears in $[M]$. By the definition of $[M], r-s \geq n-b+1$, which yields

$$
\begin{aligned}
(k-(p+1)+1)+(q+1) & \leq n-(p-q)+1 \\
& =n+(r-p)+(q-s)-(r-s)+1 \\
& \leq(r-p)+(q-s)+b .
\end{aligned}
$$

Moreover, by the maximality of $b$, we have $(r-p)+(q-s) \leq a-b+1$, which implies

$$
(k-(p+1)+1)+(q+1) \leq a+1 .
$$

Therefore, $\psi(M)$ satisfies the condition for the tableaux in $S\left(\Lambda_{k}\right)$.

Conversely, let $S$ be a tableau in $S\left(\Lambda_{k}\right)$ with the entries $i_{1}, \cdots, i_{k}$ from bottom to top. If there is no pair $\left(i_{p}=a, i_{q}=\bar{a}\right)$ such that $p-q \leq n-a$, we define $\psi^{-1}(S)$ to be the monomial

$$
X_{i_{1}}(1) X_{i_{2}}(2) \cdots X_{i_{k}}(k)
$$

If there is a pair $\left(i_{p}=a, i_{q}=\bar{a}\right)$ such that $p-q \leq n-a$, we define $\psi^{-1}(S)$ to be the monomial

$$
X_{i_{1}}(1) \cdots X_{\overline{a-1}}(q) \cdots X_{a-1}(p) \cdots X_{i_{k}}(k) .
$$

If there are several such pairs, $\psi^{-1}(S)$ is defined by applying the above rule repeatedly from $a=n-1$ to $a=2$. Then it is easy to see that $\psi^{-1}(S) \in \mathcal{M}\left(\Lambda_{k}\right)$ and that $\psi$ and $\psi^{-1}$ are inverses to each other. 
It remains to show that $\psi$ is a crystal morphism. For a monomial $M=$ $X_{i_{1}}(1) \cdots X_{i_{k}}(k)$ of $\mathcal{M}\left(\Lambda_{k}\right)$, assume that $\tilde{f}_{i} M \neq 0$. Then for some $p$ and $q$, we have $i_{p}=i$ and $i_{p-1} \neq i+1$, or $i_{q}=\overline{i+1}$ and $i_{q-1} \neq \bar{i}$. Moreover,

$$
\begin{aligned}
X_{i}(p) & =Y_{i-1}(p+1)^{-1} Y_{i}(p), \\
X_{\overline{i+1}}(q) & =Y_{i}((n-i)+q) Y_{i+1}((n-i)+q)^{-1} .
\end{aligned}
$$

If $p-q \geq n-i+1$, then $p>(n-i)+q$ and so $\tilde{f}_{i} M$ is obtained by multiplying

$$
A_{i}(p)^{-1}=X_{i}(p)^{-1} X_{i+1}(p)
$$

Since $\tilde{f}_{i} \psi(M)$ is obtained from $\psi(M)$ by replacing $i$ with $i+1$ by the tensor product rule for the Kashiwara operators, it is clear that $\psi\left(\tilde{f}_{i} M\right)=\tilde{f}_{i} \psi(M)$.

If $p-q<n-i$, then $p<(n-i)+q$, and $\tilde{f}_{i} M$ is obtained by multiplying

$$
A_{i}((n-i)+q)^{-1}=X_{\overline{i+1}}(q)^{-1} X_{\bar{i}}(q) .
$$

That is,

$$
\tilde{f}_{i} M=X_{i_{1}}(1) \cdots X_{\bar{i}}(q) \cdots X_{i}(p) \cdots X_{i_{k}}(k) .
$$

Furthermore, since $p-q<n-i, \psi\left(\tilde{f}_{i} M\right)$ is the tableau with the entries

$$
i_{1}, \cdots, i_{q}+1=\overline{i+1}, \cdots, \overline{i_{p}}+1=i+1, \cdots, i_{k} .
$$

Recall that $\tilde{f}_{i} \psi(M)$ is obtained from $\psi(M)$ by replacing $i$ with $i+1$. Therefore, $\psi\left(\tilde{f}_{i} M\right)=\tilde{f}_{i} \psi(M)$.

Similarly, we can prove $\psi\left(\tilde{e}_{i} M\right)=\tilde{e}_{i} \psi(M)$.

Theorem 3.3. Let $\lambda=a_{1} \Lambda_{1}+\cdots+a_{n} \Lambda_{n}$ be a dominant integral weight. Then there is a crystal isomorphism $\psi: \mathcal{M}(\lambda) \rightarrow S(\lambda)$.

Proof. Let $M$ be a monomial in $\mathcal{M}(\lambda)$, which can be expressed as

$$
M=\prod_{i \in \mathbf{B} ; 1 \leq j \leq n} X_{i}(j)^{m_{i j}} .
$$

Consider the tableau $T([M])$ associated with $[M]$. If there is no pair $(a(p), \bar{a}(q))$ such that

(a) $p-q<n-a$, and $a(p)$ and $\bar{a}(q)$ lie in the same column, or

(b) $\bar{a}(q)$ lies in the left-hand side of $a(p)$,

we define $\psi(M)$ to be the semistandard tableau with $m_{i j}$-many $i$ entries in the $j$-th row (from the bottom) for $i=1, \cdots, \overline{1}, j=1, \cdots, n$. By conditions for $\mathcal{M}(\lambda)$, it is well-defined. 
Secondly, suppose that there is a pair $(a(p), \bar{a}(q))$ satisfying the conditions (a) or (b) in $T([M])$. Then we define $\psi(M)$ to be the tableau obtained from $T([M])$ by replacing $a$ with $a+1$. If there are several such pairs $(a(p), \bar{a}(q))$ such that $p-q<n-a(a=1, \cdots, n-1)$, then $\psi(M)$ is defined by applying the above rule repeatedly from 1 to $n-1$.

Conversely, let $S$ be a tableau in $S(\lambda)$. If there is no pair $(a(p), \bar{a}(q))$ satisfying the conditions (a) or (b), we define $\psi^{-1}(S)$ to be the monomial $\prod X_{i}(p)$, where $X_{i}(p)$ corresponds to the entry $i$ in the $p$-th row of $S$. Suppose that there is a pair $(a(p), \bar{a}(q))$ satisfying the conditions (a) or (b). Then we have a tableau $S^{\prime}$ obtained from $S$ by replacing $a$ in the $p$-th row and $\bar{a}$ in the $q$-th row with $a-1$ and $\overline{a-1}$. If there are several such pairs, then $S^{\prime}$ is defined by applying the above rule repeatedly from $a=n$ to $a=2$. Now, we define $\psi^{-1}(S)$ to be the monomial associated to $S^{\prime}$.

Then by the same argument in Proposition 3.2, we can see that $\psi$ and $\psi^{-1}$ are inverses to each other and that $\psi$ is a crystal isomorphism.

Example 3.4. Let $\lambda=\Lambda_{1}+\Lambda_{3}$ for $C_{4}$ and let $M=Y_{1}(5)^{-2} Y_{2}(2)$, which can be expressed as

$$
[M]=X_{1}(3) X_{2}(2) X_{\overline{1}}(1)^{2}
$$

and

$$
T([M])=\begin{array}{|l|l|} 
& 1 \\
\cline { 2 - 2 } & 2 \\
\hline \overline{1} & \overline{1} \\
\hline
\end{array}
$$

Since there is a pair $(1(3), \overline{1}(1))$ in $T([M])$ such that $p-q=3-1<4-1=n-a$, they corresponds to $(2(3), \overline{2}(1))$. Here, $\overline{1}(1)$ is just the one in the second column from right. Moreover, this changed $\overline{2}(1)$ and 2(2) also satisfies $p-q=2-1<$ $4-2=n-a$, which implies that they corresponds to $(3(2), \overline{3}(1))$. Therefore,

$$
\psi(M)=\begin{array}{|l|l|}
\cline { 2 - 2 } & 2 \\
\cline { 2 - 2 } & 3 \\
\hline \overline{3} & \overline{1} \\
\hline
\end{array}
$$

\section{§3.2. $U_{q}\left(B_{n}\right)$ type}

\section{Proposition 3.5.}

(a) For $k=1, \ldots, n$, there is a crystal isomorphism $\psi: \mathcal{M}\left(\omega_{k}\right) \rightarrow S\left(\omega_{k}\right)$.

(b) There is a crystal isomorphism $\psi: \mathcal{M}\left(\Lambda_{n}\right) \rightarrow S\left(\Lambda_{n}\right)$. 
Proof. (a) The map $\psi$ is defined by the same algorithm in Proposition 3.2 and the proof is similar to that of Proposition 3.2.

(b) For a monomial $M=\sqrt{X_{i_{1}}(1) \cdots X_{i_{n}}(n)} \in \mathcal{M}\left(\Lambda_{n}\right)$, define $\psi(M)$ by the tableau of half column with entries $i_{1}, \cdots, i_{n}$ reading from bottom to top. Then by definition of $\mathcal{M}\left(\Lambda_{n}\right)$ and $S\left(\Lambda_{n}\right)$, it is easy to see that $\psi$ is a crystal isomorphism over $U_{q}\left(B_{n}\right)$.

Proposition 3.6. For $k=1, \ldots, n$, there is a crystal isomorphism $\psi$ : $\mathcal{M}\left(\omega_{k}+\Lambda_{n}\right) \rightarrow S\left(\omega_{k}+\Lambda_{n}\right)$.

Proof. Let $M$ be a monomial in $\mathcal{M}\left(\omega_{k}+\Lambda_{n}\right)$. Then $[M]$ is expressed as

$$
[M]=X_{i_{1}}(1) \cdots X_{i_{k}}(k) \sqrt{X_{j_{1}}(1) \cdots X_{j_{n}}(n)} .
$$

If there is no pair $\left(X_{a}(p), X_{\bar{a}}(q)\right)$ such that $p-q<n-a$, we define $\psi(M)$ to be the tableau consisting of one column of length $k$ and the half column of length $n$ with entries $i_{1}, \cdots, i_{k}$ and $j_{1}, \cdots, j_{n}$ from bottom to top and from left to right. Otherwise, $\psi(M)$ is defined to be the tableau with entries $i_{1}, \cdots, \overline{a+1}, \cdots, a+$ $1, \cdots, i_{k}$ and $j_{1}, \cdots, j_{n}$.

Then by definition of $[M]$ and $\psi$, it is clear that the tableau $\psi(M)$ is semistandard. Moreover, by the condition of $S\left(\Lambda_{n}\right)$, it is easy to see that $\psi(M)$ satisfies the conditions for the tableaux in $S\left(\omega_{k}+\Lambda_{n}\right)$.

Conversely, let $T$ be a tableau in $S\left(\omega_{k}+\Lambda_{n}\right)$ with entries $i_{1}, \cdots, i_{k}$ and $j_{1}, \cdots, j_{n}$ from bottom to top and from left to right. If there is no pair $\left(i_{p}=\right.$ $\left.a, i_{q}=\bar{a}\right)$ such that $p-q \leq n-a$, we define $\psi^{-1}(T)$ to be the monomial

$$
X_{i_{1}}(1) \cdots X_{i_{k}}(k) \sqrt{X_{j_{1}}(1) \cdots X_{j_{n}}(n)} .
$$

If there is a pair $\left(i_{p}=a, i_{q}=\bar{a}\right)$ such that $q-p \leq n-a$, we define $\psi^{-1}(T)$ to be the monomial

$$
X_{i_{1}}(1) \cdots X_{\overline{a-1}}(q) \cdots X_{a-1}(p) \cdots X_{i_{k}}(k) \sqrt{X_{j_{1}}(1) \cdots X_{j_{n}}(n)} .
$$

Then it is clear that $\psi^{-1}(T) \in \mathcal{M}\left(\omega_{k}+\Lambda_{n}\right)$. Furthermore, $\psi$ and $\psi^{-1}$ are inverses to each other.

It remains to show that $\psi$ is a crystal morphism. If there is no $\sqrt{X_{i}(p) X_{\overline{i+1}}(q)}$ in $[M]$, it is clear that $\psi\left(\tilde{f}_{i} M\right)=\tilde{f}_{i} \psi(M)$ by Proposition 3.5 (a). Thus we may assume that there is a $\sqrt{X_{i}(p) X_{\overline{i+1}}(q)}$ in $[M]$ with $p-q=n-i-1$. Then $X_{i}(m)$ and $X_{\overline{i+1}}(l)$ exist in $[M]$ only for $m \leq p$ and 
$l \leq q$. If $m<p$ and $l<q$, it is clear that $\psi\left(\tilde{f}_{i} M\right)=\tilde{f}_{i} \psi(M)$. If there is an $X_{i}(p)$ in $[M]$, then $\tilde{f}_{i} M$ is obtained by multiplying

$$
X_{i}(p)^{-1} X_{i+1}(p)=\sqrt{X_{i}(p)^{-1} X_{\overline{i+1}}(q)^{-1} X_{i+1}(p) X_{\bar{i}}(q)} .
$$

Therefore, $\psi\left(\tilde{f}_{i} M\right)=\tilde{f}_{i} \psi(M)$. Similarly, if there is an $X_{\overline{i+1}}(q)$ in $[M]$, we have $\psi\left(\tilde{f}_{i} M\right)=\tilde{f}_{i} \psi(M)$.

Theorem 3.7. Let $\lambda=a_{1} \omega_{1}+\cdots+a_{n} \omega_{n}+b \Lambda_{n}(b=0$ or 1$)$. Then there is a crystal isomorphism $\psi: \mathcal{M}(\lambda) \rightarrow S(\lambda)$.

Proof. Combining Proposition 3.6 and the argument in Theorem 3.3, we obtain the desired result.

Example 3.8. Let $\lambda=\omega_{2}+\omega_{3}+\Lambda_{4}$ for $B_{4}$ and let

$$
M=Y_{1}(3)^{-1} Y_{1}(4) Y_{1}(5)^{-2} Y_{2}(2)^{2} Y_{2}(4)^{-1} Y_{4}(2) \text {. }
$$

Then

$$
[M]=X_{1}(3) X_{2}(2)^{2} X_{\overline{1}}(1) X_{\overline{2}}(1) \sqrt{X_{2}(4) X_{3}(3) X_{4}(2) X_{\overline{1}}(1)}
$$

and

$$
T([M])=\begin{array}{|l|l|l|}
\cline { 3 - 4 } & 1 & 3 \\
\hline 2 & 2 & 4 \\
\hline \overline{2} & \overline{1} & \overline{1} \\
\hline
\end{array}
$$

If we consider the pairs $(1(3), \overline{1}(1))$ and $(2(2), \overline{2}(1))$ (Of course, $2(2)$ is just one in the second column.), then we have $3-1<4-1=n-a$ and $2-1<4-2=n-a$. Therefore,

$$
\psi(M)=\begin{array}{|l|l|l|}
\cline { 3 - 3 } & 2 & 3 \\
\hline 2 & 3 & 4 \\
\hline \overline{3} & \overline{2} & \overline{1} \\
\hline
\end{array}
$$

$\S 3.3 . \quad U_{q}\left(D_{n}\right)$ type

Proposition 3.9. (a) For $k=1, \cdots, n+1$, there is a crystal isomorphism $\psi: \mathcal{M}\left(\omega_{k}\right) \rightarrow S\left(\omega_{k}\right)$.

(b) For $\lambda=\Lambda_{n}$ or $\Lambda_{n-1}$, there is a crystal isomorphism $\psi: \mathcal{M}(\lambda) \rightarrow S(\lambda)$.

(c) For $k=1, \cdots, n$, there is a crystal isomorphism $\psi: \mathcal{M}\left(\omega_{k}+\Lambda_{n}\right) \rightarrow$ $S\left(\omega_{k}+\Lambda_{n}\right)$.

(d) For $k=1, \cdots, n-1, n+1$, there is a crystal isomorphism $\psi: \mathcal{M}\left(\omega_{k}+\right.$ $\left.\Lambda_{n-1}\right) \rightarrow S\left(\omega_{k}+\Lambda_{n-1}\right)$. 
Proof. (a) Let $[M]=X_{i_{1}}(1) \cdots X_{i_{k}}(k)$ be a monomial of $\mathcal{M}\left(\omega_{k}\right)$. If there is no pair $\left(X_{a}(p), X_{\bar{a}}(q)\right)$ such that $p-q<(n-1)-a$, we define $\psi(M)$ to be the tableau of one column with entries $i_{1}, \cdots, i_{k}$ from bottom to top.

Suppose that there is a pair $\left(X_{a}(p), X_{\bar{a}}(q)\right)$ such that $p-q<(n-$ $1)-a$. Then we define $\psi(M)$ to be the tableau of one column with entries $i_{1}, \cdots, \overline{a+1}, \cdots, a+1, \cdots, i_{k}$ from bottom to top. If there are several such pairs $\left(X_{a}(p), X_{\bar{a}}(q)\right)$ such that $p-q<(n-1)-a(a=1, \cdots, n)$, then $\psi(M)$ is defined by applying the above rule repeatedly from 1 to $n$.

Then by the same argument in the proof of Proposition 3.2, we see that $\psi$ is a crystal isomorphism.

(b) The map $\psi$ is defined by the same way as in Proposition 3.6. It is clear that $\psi$ is a crystal isomorphism.

(c),(d) The proof is similar to that of Proposition 3.5.

Theorem 3.10. Let $\lambda$ be dominant integral weight. Then there is a crystal isomorphism $\psi: \mathcal{M}(\lambda) \rightarrow S(\lambda)$.

Proof. Combining Proposition 3.9 and the argument in Theorem 3.3, we get the desired result.

Example 3.11. Let $\lambda=2 \omega_{2}+\Lambda_{3}$ for $D_{4}$ and let

$$
M=Y_{1}(2)^{2} Y_{1}(4)^{-3} Y_{4}(2) .
$$

Then

$$
[M]=X_{1}(2)^{2} X_{\overline{1}}(1)^{2}\left|\sqrt{X_{2}(4) X_{3}(3) X_{4}(2) X_{\overline{1}}(1)}\right|
$$

and

$$
T([M])=\begin{array}{|l|l|l|l|}
\cline { 3 - 4 } & & 3 \\
\hline 1 & 1 & 4 \\
\hline \overline{1} & \overline{1} & \overline{1} \\
\hline
\end{array}
$$

Furthermore, if we consider $1(2)$ of the second column and $\overline{1}(1)$ of the third column from right to left, then $p-q=2-1<(4-1)-1=(n-1)-a$. Therefore,

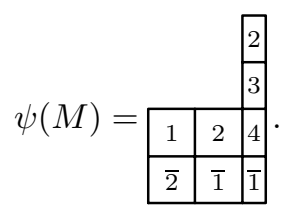


Finally, we close this section with a bijection between the monomial realization and the Kashiwara-Nakashima tableau realization.

Proposition $3.12[9,10]$. For a dominant integral weight $\lambda$, there is a $U_{q}(\mathfrak{g})$-crystal isomorphism $\varphi: S(\lambda) \rightarrow T(\lambda)$ for $\left(\mathfrak{g}=A_{n}, C_{n}, B_{n}, D_{n}\right)$ given by

$$
\varphi(S)=S_{1} \leftarrow S_{2} \leftarrow \cdots \leftarrow S_{t},
$$

where $S_{i}$ is the $i$-th column of $S$ from right to left.

Corollary 3.13. Let $\lambda$ be a dominant integral weight. There is a $U_{q}(\mathfrak{g})$ crystal isomorphism $\phi: \mathcal{M}(\lambda) \rightarrow T(\lambda)$ for $\mathfrak{g}=A_{n}, C_{n}, B_{n}, D_{n}$.

Proof. By Theorem 3.3, Theorem 3.7, Theorem 3.10 and Proposition 3.12, $\phi=\varphi \circ \psi$ is the desired crystal isomorphism.

Example 3.14. Let $\lambda=\Lambda_{1}+2 \Lambda_{2}+\Lambda_{3}$ for $C_{3}$ and let $M=$ $Y_{1}(2)^{2} Y_{1}(3) Y_{1}(4)^{-2} Y_{2}(3)^{-1} Y_{3}(1)$. Then

$$
[M]=X_{2}(3) X_{3}(2) X_{1}(2)^{2} X_{\overline{1}}(1) X_{\overline{2}}(1) X_{\overline{3}}(1) X_{3}(1)
$$

and

$$
T([M])=\psi(M)=\begin{array}{|l|l|l|l|}
\cline { 3 - 4 } & 1 & 1 & 3 \\
\hline 3 & \overline{3} & \overline{2} & \overline{1} \\
\hline
\end{array}
$$

It follows that

$$
\begin{aligned}
& \phi(M)=\varphi(\psi(M))=\frac{\mid \begin{array}{l|}
2 \\
\hline 3 \\
\hline \overline{1}
\end{array}}{5} \leftarrow \begin{array}{|l|}
\hline 1 \\
\hline \overline{2} \\
\hline
\end{array} \leftarrow \begin{array}{|l|}
\hline 1 \\
\hline \overline{3} \\
\hline
\end{array} \\
& =\begin{array}{|l|l|l|l|}
\hline 1 & 1 & 2 & \overline{1} \\
\hline 2 & \overline{2} & \overline{2} & \\
\cline { 1 - 3 } 3 & \multicolumn{3}{|c}{} \\
\cline { 1 - 1 }
\end{array} \text {. }
\end{aligned}
$$

Conversely, let

$$
T=\begin{array}{l|l|l|l|}
\hline 1 & 1 & 2 & \overline{1} \\
\hline 2 & \overline{2} & \overline{2} &
\end{array} \in T\left(\Lambda_{1}+2 \Lambda_{2}+\Lambda_{3}\right) .
$$


By applying the reverse bumping rule to the entries from bottom to top and from right to left $[9,10]$, we have

$$
\varphi^{-1}(T)=\begin{array}{|l|l|l|l|}
\cline { 3 - 4 } & 1 & 1 & 3 \\
\hline 3 & \overline{3} & \overline{2} & \overline{1} \\
\hline
\end{array}
$$

Therefore, we have

$$
\begin{aligned}
\phi^{-1}(T)=\psi^{-1}\left(\varphi^{-1}(T)\right) & =X_{2}(3) X_{1}(2)^{2} X_{3}(2) X_{3}(1) X_{\overline{3}}(1) X_{\overline{2}}(1) X_{\overline{1}}(1) \\
& =Y_{1}(2)^{2} Y_{1}(3) Y_{1}(4)^{-2} Y_{2}(3)^{-1} Y_{3}(1) .
\end{aligned}
$$

\section{References}

[1] Hong, J. and Kang, S.-J., Introduction to Quantum Groups and Crystal Bases, Grad. Stud. Math., 42, Amer. Math. Soc., 2002.

[2] Kang, S.-J., Crystal bases for quantum affine algebras and combinatorics of Young walls, Proc. London Math. Soc., 86 (2003), 29-69.

[3] Kang, S.-J., Kim, J.-A., Lee, H. and Shin, D.-U., Young wall realization of crystal bases for classical Lie algebras, Trans. Amer. Math. Soc., 356 (2004), 2349-2378.

[4] Kang, S.-J., Kim, J.-A. and Shin, D.-U., Monomial realization of crystal bases for special linear Lie algebras, J. Algebra, 274 (2004), 629-642.

[5] Kashiwara, M., On crystal bases of the $q$-analogue of universal enveloping algebras, Duke Math. J., 63 (1991), 465-516.

[6] , Realizations of crystals, in Combinatorial and Geometric Representation Theory (Seoul, 2001), Kang, S.-J. and Lee, K.-H., (eds.), Contemp. Math., 325 (2003), 133-139, Amer. Math. Soc.

[7] Kashiwara, M. and Nakashima, T., Crystal graphs for representations of the $q$-analogue of classical Lie algebras, J. Algebra, 165 (1994), 295-345.

[8] Kim, J.-A., Monomial realization of crystal graphs for $U_{q}\left(A_{n}^{(1)}\right)$, submitted.

[9] Kim, J.-A. and Shin, D.-U., Insertion scheme for the classical Lie algebras, to appear in Comm. Algebra.

[10] Correspondence between Young walls and Young tableaux realizations of crystal bases for the classical Lie algebras, Preprint math.QA/0303287, submitted.

[11] Nakajima, H., Quiver varieties and tensor products, Invent. Math., 146 (2001), 399-449.

[12] t-analogs of q-characters of quantum affine algebras of type $A_{n}, D_{n}$, in Combinatorial and Geometric Representation Theory (Seoul, 2001), Kang, S.-J. and Lee, K.H.,(eds.), Contemp. Math., 325 (2003), 141-160, Amer. Math. Soc. 